\title{
Can $\mathcal{B}\left(\ell^{p}\right)$ ever be amenable?
}

\author{
by \\ Matthew Daws (Leeds) and Volker Runde (Edmonton)
}

\begin{abstract}
It is known that $\mathcal{B}\left(\ell^{p}\right)$ is not amenable for $p=1,2, \infty$, but whether or not $\mathcal{B}\left(\ell^{p}\right)$ is amenable for $p \in(1, \infty) \backslash\{2\}$ is an open problem. We show that, if $\mathcal{B}\left(\ell^{p}\right)$ is amenable for $p \in(1, \infty)$, then so are $\ell^{\infty}\left(\mathcal{B}\left(\ell^{p}\right)\right)$ and $\ell^{\infty}\left(\mathcal{K}\left(\ell^{p}\right)\right)$. Moreover, if $\ell^{\infty}\left(\mathcal{K}\left(\ell^{p}\right)\right)$ is amenable so is $\ell^{\infty}(\mathbb{I}, \mathcal{K}(E))$ for any index set $\mathbb{I}$ and for any infinite-dimensional $\mathcal{L}^{p}$-space $E$; in particular, if $\ell^{\infty}\left(\mathcal{K}\left(\ell^{p}\right)\right)$ is amenable for $p \in(1, \infty)$, then so is $\ell^{\infty}\left(\mathcal{K}\left(\ell^{p} \oplus \ell^{2}\right)\right)$. We show that $\ell^{\infty}\left(\mathcal{K}\left(\ell^{p} \oplus \ell^{2}\right)\right)$ is not amenable for $p=1, \infty$, but also that our methods fail us if $p \in(1, \infty)$. Finally, for $p \in(1,2)$ and a free ultrafilter $\mathcal{U}$ over $\mathbb{N}$, we exhibit a closed left ideal of $\left(\mathcal{K}\left(\ell^{p}\right)\right) \mathcal{U}$ lacking a right approximate identity, but enjoying a certain very weak complementation property.
\end{abstract}

Introduction. In his seminal memoir [Joh 1], B. E. Johnson initiated the theory of amenable Banach algebras. The choice of terminology is motivated by [Joh 1, Theorem 2.5]: a locally compact group $G$ is amenable in the usual sense (see [Pat], for instance) if and only if its group algebra $L^{1}(G)$ is an amenable Banach algebra.

Ever since [Joh 1] was published, there have been ongoing efforts to determine, for particular classes of Banach algebras, which algebras in them are the amenable ones. One spectacular result in this direction is the characterization of the amenable $C^{*}$-algebras: a $C^{*}$-algebra is amenable if and only if it is nuclear (this result, mostly credited to A. Connes and U. Haagerup, is the culmination of the efforts of many mathematicians; see [Run] or [Tak] for self-contained accounts).

One particular class of Banach algebras for which the problem of characterizing its amenable members is still wide open is the class of Banach algebras $\mathcal{B}(E)$, the algebras of all bounded linear operators on a Banach space $E$. From a philosophical point of view, this problem ought to be easy: amenability can often be thought of as a weak finiteness condition, and, for

2000 Mathematics Subject Classification: Primary 47L10; Secondary 46B07, 46B08, 46B45, 46E30, 46H20, 47L20.

Key words and phrases: amenability, $\mathcal{L}^{p}$-spaces, maximal operator ideals, ultraamenability.

Research of the second-named author supported by NSERC. 
any infinite-dimensional Banach space $E$, the algebra $\mathcal{B}(E)$ should simply be too "large" to be amenable. Already Johnson asked in [Joh 1]:

- Is $\mathcal{B}(E)$ ever amenable for infinite-dimensional $E$ ? ([Joh 1, 10.4])

- Is $\mathcal{B}(\mathfrak{H})$ amenable for an infinite-dimensional Hilbert space $\mathfrak{H}$ ? ([Joh 1, 10.2])

The Hilbert space case was settled relatively quickly: in [Was], S. Wassermann showed that a nuclear von Neumann algebra had to be subhomogeneous. In view of the equivalence of amenability and nuclearity for $C^{*}$ algebras, this means that $\mathcal{B}(\mathfrak{H})$ can be amenable only if $\operatorname{dim} \mathfrak{H}<\infty$.

Ever since, very little progress has been made in the general Banach space case. Until recently, it was not even known whether $\mathcal{B}\left(\ell^{p}\right)$ was amenable or not for any $p \in[1, \infty]$ other than 2 . This situation changed with C. J. Read's paper [Rea]: making ingenious use of random hypergraphs, Read showed that $\mathcal{B}\left(\ell^{1}\right)$ is not amenable. Moreover, he showed that, for any $p \in[1, \infty] \backslash\{2\}$, the Banach algebra $\ell^{\infty}-\bigoplus_{n=1}^{\infty} \mathcal{B}\left(\ell_{n}^{p}\right)$ also fails to be amenable (the $p=2$ case already follows from Wassermann's result). Subsequently, G. Pisier simplified Read's proof by replacing the random hypergraphs of [Rea] with expanders ([Pis]). Eventually, N. Ozawa simplified Pisier's argument even further and succeeded in giving a proof that simultaneously established the non-amenability of $\mathcal{B}\left(\ell^{p}\right)$ for $p=1,2, \infty$ and of $\ell^{\infty}-\bigoplus_{n=1}^{\infty} \mathcal{B}\left(\ell_{n}^{p}\right)$ for any $p \in[1, \infty]$ ([Oza]); even though it is not explicitly stated in [Oza], the proof also works for $\mathcal{B}\left(c_{0}\right)$.

In the present paper, we investigate what consequences the hypothetical amenability of $\mathcal{B}\left(\ell^{p}\right)$ for $p \in(1, \infty) \backslash\{2\}$ would have.

Our first result is that, if $\mathcal{B}\left(\ell^{p}\right)$ is amenable, then so is $\ell^{\infty}\left(\mathcal{B}\left(\ell^{p}\right)\right)$. As the much "smaller" algebra $\ell^{\infty}-\bigoplus_{n=1}^{\infty} \mathcal{B}\left(\ell_{n}^{p}\right)$ is not amenable, this lends again support to the belief that $\mathcal{B}\left(\ell^{p}\right)$ is not amenable (even though, of course, this is a far cry from a proof).

A straightforward consequence of the amenability of $\ell^{\infty}\left(\mathcal{B}\left(\ell^{p}\right)\right)$ is that $\ell^{\infty}\left(\mathcal{K}\left(\ell^{p}\right)\right)$ is amenable as well, and we shall devote most of this paper to exploring the consequences of the amenability of that particular Banach algebra and, more generally, of $\ell^{\infty}(\mathcal{K}(E))$ for particular Banach spaces $E$. (Incidentally, the question of whether $\ell^{\infty}(\mathcal{K}(E))$ is amenable for specific Banach spaces $E$ seems to have received almost no attention in the literature; the only references known to the authors are [CS-R] and [L-L-W], where the case $E=\ell^{2}$ is settled in the negative.)

First, we show that, due to the separability of $\mathcal{K}\left(\ell^{p}\right)$, the amenability of $\ell^{\infty}\left(\mathcal{K}\left(\ell^{p}\right)\right)$ already entails the amenability of $\ell^{\infty}\left(\mathbb{I}, \mathcal{K}\left(\ell^{p}\right)\right)$ for every index set $\mathbb{I}$ and thus of $\left(\mathcal{K}\left(\ell^{p}\right)\right) \mathcal{U}$ for every ultrafilter $\mathcal{U}$ (so that $\mathcal{K}\left(\ell^{p}\right)$ is ultra-amenable in the terminology of [Daw 2]). 
Next, we see that the amenability of $\ell^{\infty}\left(\mathcal{K}\left(\ell^{p}\right)\right)$ forces $\ell^{\infty}(\mathbb{I}, \mathcal{K}(E))$ to be amenable for every index set $\mathbb{I}$ and every infinite-dimensional $\mathcal{L}^{p}$-space $E$ in the sense of [L-P]. In particular, if $\ell^{\infty}\left(\mathcal{K}\left(\ell^{p}\right)\right)$ is amenable, the so is $\ell^{\infty}\left(\mathcal{K}\left(\ell^{p} \oplus \ell^{2}\right)\right)$, which is interesting because $\mathcal{B}\left(\ell^{p} \oplus \ell^{2}\right)$ is known to be nonamenable.

We then study the amenability of $\ell^{\infty}(\mathcal{K}(E \oplus F))$ for certain Banach spaces $E$ and $F$. Using the theory of operator ideals (see [Pie]), we show that $\ell^{\infty}\left(\mathcal{K}\left(E \oplus \ell^{2}\right)\right)$ is not amenable for $E=c_{0}, \ell^{\infty}$, and $\ell^{1}$, but we also show that our methods fail to establish the non-amenability of $\ell^{\infty}\left(\mathcal{B}\left(\ell^{p} \oplus \ell^{2}\right)\right)$ for $p \in(1, \infty)$.

Finally, we take a look at a particular left ideal of $\left(\mathcal{K}\left(\ell^{p}\right)\right) \mathcal{U}$ for $p \in(1,2)$ and $\mathcal{U}$ a free ultrafilter over $\mathbb{N}$. We show that this ideal lacks a right approximate identity and, at the same time, enjoys a certain complementation property, which is unfortunately too weak to obtain a contradiction to the amenability of $\left(\mathcal{K}\left(\ell^{p}\right)\right) \mathcal{U}$.

Acknowledgments. This research was initiated while the first author was visiting the University of Alberta in the summer of 2007; the financial support and the kind hospitality are gratefully acknowledged. Both authors would like to thank Andreas Defant, Albrecht Pietsch, and Nicole TomczakJaegermann for valuable help with operator ideals.

1. Amenable Banach algebras. The definition of an amenable Banach algebra given in [Joh 1] is in terms of certain derivations being inner. Throughout this paper, however, we shall not rely on that definition directly, but rather on a more intrinsic, but equivalent characterization, also due to Johnson ([Joh 2]).

Let $\mathfrak{A}$ be a Banach algebra, and let $E$ and $F$ be a left and right Banach $\mathfrak{A}$-module, respectively. We use $\hat{\otimes}$ to denote the projective tensor product of Banach spaces. The Banach space $E \hat{\otimes} F$ becomes a Banach $\mathfrak{A}$-bimodule via $a \cdot(x \otimes y):=a \cdot x \otimes y \quad$ and $\quad(x \otimes y) \cdot a:=x \otimes y \cdot a \quad(a \in \mathfrak{A}, x \in E, y \in F)$.

In particular, $\mathfrak{A} \hat{\otimes} \mathfrak{A}$ is a Banach $\mathfrak{A}$-bimodule in a canonical manner. With respect to these module operatations, the diagonal map $\Delta: \mathfrak{A} \hat{\otimes} \mathfrak{A} \rightarrow \mathfrak{A}$ induced by multiplication, i.e., $\Delta(a \otimes b)=a b$ for $a, b \in \mathfrak{A}$, is a bimodule homomorphism; if we want to emphasize the algebra $\mathfrak{A}$, we sometimes write $\Delta_{\mathfrak{A}}$ for $\Delta$.

Definition 1.1. Let $\mathfrak{A}$ be a Banach algebra. An approximate diagonal for $\mathfrak{A}$ is a bounded net $\left(\boldsymbol{d}_{\alpha}\right)_{\alpha}$ in $\mathfrak{A} \hat{\otimes} \mathfrak{A}$ such that

$$
a \cdot \boldsymbol{d}_{\alpha}-\boldsymbol{d}_{\alpha} \cdot a \rightarrow 0 \quad(a \in \mathfrak{A})
$$


and

$$
a \Delta \boldsymbol{d}_{\alpha} \rightarrow a \quad(a \in \mathfrak{A}) .
$$

If $\mathfrak{A}$ has an approximate diagonal bounded, we say that $\mathfrak{A}$ is amenable.

REMARKS.

1. If $\mathfrak{A}$ is amenable and has an identity $1_{\mathfrak{A}}$, then there is an approximate diagonal $\left(\boldsymbol{d}_{\alpha}\right)_{\alpha \in \mathbb{A}}$ for $\mathfrak{A}$ such that $\Delta \boldsymbol{d}_{\alpha}=1_{\mathfrak{A}}$ for all $\alpha \in \mathbb{A}$.

2. If $\mathfrak{A}$ is amenable with an approximate diagonal bounded by $C \geq 1$, then $\mathfrak{A}$ is also called $C$-amenable. It is clear from (2) that it does not make sense to speak of $C$-amenability for any $C \in(0,1)$.

For modern accounts of the theory of amenable Banach algebras, see [Dal] or [Run].

The hereditary properties of Banach-algebraic amenability are well understood. For instance, quotients of amenable Banach algebras are again amenable ([Run, Corollary 2.3.2]), and a closed ideal of an amenable Banach algebra is amenable if and only if it has a bounded approximate identity and if and only if it is weakly complemented ([Run, Theorem 2.3.7]). Whether or not a particular subalgebra of an amenable Banach algebra is amenable is a much more delicate question and an elegant characterization is certainly out of reach. Nevertheless, some partial results exist such as the following ([G-J-W, Theorem 6.2]):

THEOREM 1.2. Let $\mathfrak{A}$ be a Banach algebra with a bounded approximate identity, let $P_{1} \in \mathcal{M}(\mathfrak{A})$ be a projection, and let $P_{2}:=\mathrm{id}_{\mathfrak{A}}-P_{1}$. Suppose further that $\Delta_{\mathfrak{A}}$ maps $P_{2} \mathfrak{A} P_{1} \hat{\otimes} P_{1} \mathfrak{A} P_{2}$ onto $P_{2} \mathfrak{A} P_{2}$. Then $\mathfrak{A}$ is amenable if and only if $P_{1} \mathfrak{A} P_{1}$ is amenable.

Here, $\mathcal{M}(\mathfrak{A})$ stands for the multiplier algebra of $\mathfrak{A}$ ([Dal, p. 60]).

The Banach algebra $\mathfrak{A}$ in Theorem 1.2 has a matrix-like structure thanks to the projections $P_{1}$ and $P_{2}$. We shall now prove a necessary condition for the non-amenability of such algebras:

Proposition 1.3. Let $\mathfrak{A}$ be a Banach algebra, let $P_{1} \in \mathcal{M}(\mathfrak{A})$ be an idempotent, and let $P_{2}:=\mathrm{id}_{\mathfrak{A}}-P_{1}$. Suppose that there is a closed ideal I of $\mathfrak{A}$ such that $P_{2} I P_{1}=P_{2} \mathfrak{A} P_{1}$, but $P_{1} I P_{2} \subsetneq P_{1} \mathfrak{A} P_{2}$. Then $\mathfrak{A}$ is not amenable.

Proof. For $j, k \in\{1,2\}$, set

$$
\mathfrak{A}_{j, k}:=P_{j} \mathfrak{A} P_{k} \quad \text { and } \quad I_{j, k}:=P_{j} I P_{k} .
$$

It follows that

$$
\mathfrak{A} \cong\left[\begin{array}{ll}
\mathfrak{A}_{1,1} & \mathfrak{A}_{1,2} \\
\mathfrak{A}_{2,1} & \mathfrak{A}_{2,2}
\end{array}\right] \quad \text { and } \quad I \cong\left[\begin{array}{cc}
I_{1,1} & I_{1,2} \\
I_{2,1} & I_{2,2}
\end{array}\right]
$$

and thus 


$$
\mathfrak{A} / I \cong\left[\begin{array}{ll}
\mathfrak{A}_{1,1} / I_{1,1} & \mathfrak{A}_{1,2} / I_{1,2} \\
\mathfrak{A}_{2,1} / I_{2,1} & \mathfrak{A}_{2,2} / I_{2,2}
\end{array}\right]=\left[\begin{array}{cc}
\mathfrak{A}_{1,1} / I_{1,1} & \mathfrak{A}_{1,2} / I_{1,2} \\
0 & \mathfrak{A}_{2,2} / I_{2,2}
\end{array}\right] .
$$

Consequently, $\left[\begin{array}{cc}0 & \mathfrak{A}_{1,2} / I_{1,2} \\ 0 & 0\end{array}\right]$ is a non-zero, complemented, nilpotent ideal of $\mathfrak{A} / I$, which is impossible if $\mathfrak{A} / I$ is amenable.

Remark. The idea behind Proposition 1.3 is implicitly already contained in [Grø, Question 4], where it is attributed to G. A. Willis. It can be used to establish the non-amenability of $\mathcal{B}(E \oplus F)$ if $\mathcal{B}(F, E)=\mathcal{K}(F, E)$, but $\mathcal{B}(E, F) \subsetneq \mathcal{K}(E, F)$; this applies, for instance, to $\mathcal{B}\left(\ell^{p} \oplus \ell^{q}\right)$ with $1 \leq p<$ $q<\infty$ ([L-T, Proposition 2.c.3]).

We conclude this section with the discussion of a stronger variant of amenability also introduced by Johnson ([Joh 3]).

Given a Banach algebra $\mathfrak{A}$, let $\Sigma$ denote the flip map on $\mathfrak{A} \hat{\otimes} \mathfrak{A}$, i.e., $\Sigma(a \otimes b)=b \otimes a$ for $a, b \in \mathfrak{A}$. An element $\boldsymbol{a} \in \mathfrak{A} \otimes \mathfrak{A}$ is called symmetric if $\Sigma \boldsymbol{a}=\boldsymbol{a}$; somewhat abusing terminology, we will also call a net in $\mathfrak{A} \otimes \mathfrak{A}$ symmetric if it consists of symmetric elements of $\mathfrak{A} \hat{\otimes} \mathfrak{A}$.

DEFinition 1.4. A Banach algebra is called symmetrically amenable if it has a symmetric approximate diagonal.

REMARK. The group algebra $L^{1}(G)$ of a locally compact group $G$ is symmetrically amenable if and only if it is amenable ([Joh 3, Theorem 4.1]) whereas the Cuntz algebras $\mathcal{O}_{n}$ for $n \in \mathbb{N}, n \geq 2$ ([Cun]) are amenable, but not symmetrically amenable ([Joh 3, p. 457]).

In view of how difficult it is, even for very well behaved Banach spaces $E$, to show that $\mathcal{B}(E)$ is not amenable, it is somewhat surprising to see how easily the corresponding question for symmetric amenability can be settled in the negative for a large class of Banach spaces:

Proposition 1.5. Let $E$ be a Banach space such that $E \cong E \oplus E$. Then $\mathcal{B}(E)$ is not symmetrically amenable.

Proof. Assume that $\mathcal{B}(E)$ is symmetrically amenable. By [Joh 3, Corollary 2.5], there is $\phi \in \mathcal{B}(E)^{*}$ such that $\left\langle\operatorname{id}_{E}, \phi\right\rangle=1$ and $\langle S T, \phi\rangle=\langle T S, \phi\rangle$ for $S, T \in \mathcal{B}(E)$.

For $j=1,2$, let $P_{j}: E \oplus E \rightarrow E$ be the projection onto the $j$ th summand. Since $E \cong E \oplus E$, there are $U_{j}, V_{j} \in \mathcal{B}(E)$ with

$$
U_{j} V_{j}=\operatorname{id}_{E} \quad \text { and } \quad V_{j} U_{j}=P_{j} \quad(j=1,2) .
$$

It follows that

$$
\begin{aligned}
1=\left\langle\operatorname{id}_{E}, \phi\right\rangle=\left\langle P_{1}+P_{2}, \phi\right\rangle & =\left\langle V_{1} U_{1}, \phi\right\rangle+\left\langle V_{2} U_{2}, \phi\right\rangle \\
& =\left\langle U_{1} V_{1}, \phi\right\rangle+\left\langle U_{2} V_{2}, \phi\right\rangle=2\left\langle\operatorname{id}_{E}, \phi\right\rangle=2,
\end{aligned}
$$

which is nonsense. 
2. Amenability of $\mathcal{B}\left(\ell^{p}\right)$ and $\ell^{\infty}\left(\mathcal{K}\left(\ell^{p}\right)\right)$. We begin by establishing some notation, part of which was already used in the introduction.

Let $\mathbb{I}$ be any index set, and let $\left(E_{i}\right)_{i \in \mathbb{I}}$ be a family of Banach spaces; we write $\prod_{i \in \mathbb{I}} E_{i}$ for its Cartesian product. For $p \in[1, \infty)$, we set

$$
\ell^{p}-\bigoplus_{i \in \mathbb{I}} E_{i}:=\left\{\left(x_{i}\right)_{i \in \mathbb{I}} \in \prod_{i \in \mathbb{I}} E_{i}: \sum_{i \in \mathbb{I}}\left\|x_{i}\right\|^{p}<\infty\right\} ;
$$

it is a linear space which becomes a Banach space if equipped with the norm

$$
\left\|\left(x_{i}\right)_{i \in \mathbb{I}}\right\|_{p}:=\left(\sum_{i \in \mathbb{I}}\left\|x_{i}\right\|^{p}\right)^{1 / p} \quad\left(\left(x_{i}\right)_{i \in \mathbb{I}} \in \ell^{p}-\bigoplus_{i \in \mathbb{I}} E_{i}\right) .
$$

Furthermore, we define

$$
\ell^{\infty}-\bigoplus_{i \in \mathbb{I}} E_{i}:=\left\{\left(x_{i}\right)_{i \in \mathbb{I}} \in \prod_{i \in \mathbb{I}} E_{i}: \sup _{i \in \mathbb{I}}\left\|x_{i}\right\|<\infty\right\} ;
$$

it, too, becomes a Banach space with the norm

$$
\left\|\left(x_{i}\right)_{i \in \mathbb{I}}\right\|_{\infty}:=\sup _{i \in \mathbb{I}}\left\|x_{i}\right\| \quad\left(\left(x_{i}\right)_{i \in \mathbb{I}} \in \ell^{\infty}-\bigoplus_{i \in \mathbb{I}} E_{i}\right) .
$$

We use $c_{0}-\bigoplus_{i \in \mathbb{I}} E_{i}$ to denote the closure of those $\left(x_{i}\right)_{i \in \mathbb{I}} \in \ell^{\infty}-\bigoplus_{i \in \mathbb{I}} E_{i}$ for which $x_{i}=0$ for all but finitely many $i \in \mathbb{I}$. We note that, if $\left(\mathfrak{A}_{i}\right)_{i \in \mathbb{I}}$ is a family of Banach algebras, then $\ell^{\infty}-\bigoplus_{i \in \mathbb{I}} \mathfrak{A}_{i}$ is a Banach algebra (which contains $c_{0}-\bigoplus_{i \in \mathbb{I}} \mathfrak{A}_{i}$ as a closed ideal). If $E_{i}=E$ for all $i \in \mathbb{I}$, we simply write $\ell^{p}(\mathbb{I}, E)$ or $c_{0}(\mathbb{I}, E)$ instead of $\ell^{p}-\bigoplus_{i \in \mathbb{I}} E$ and $c_{0}-\bigoplus_{i \in \mathbb{I}} E$, respectively. We apply the usual conventions: if $E_{i}=\mathbb{C}$ for all $i \in \mathbb{I}$ or $\mathbb{I}=\mathbb{N}$, we suppress the symbol for the space or the index set, respectively. For instance, if $p \in[1, \infty]$ and $E$ is any Banach space, then $\ell^{p}(E)$ stands for $\ell^{p}(\mathbb{N}, E)$, and if $\mathbb{I}$ is any index set, then $c_{0}(\mathbb{I})$ means $c_{0}(\mathbb{I}, \mathbb{C})$. Also, we write $\ell_{n}^{p}$ instead of $\ell^{p}(\{1, \ldots, n\}, \mathbb{C})$. Finally, for $i \in \mathbb{I}$, we let $\delta_{i}: \mathbb{I} \rightarrow \mathbb{C}$ denote the point mass at $i$; it is clear that $\delta_{i} \in \ell^{p}(\mathbb{I})$ for any $p \in[1, \infty]$.

Given any Banach space $E$, we have isometric isomorphisms between $\ell^{p}\left(\ell^{p}(E)\right)=\ell^{p}\left(\mathbb{N}^{2}, E\right)$ and $\ell^{p}(E)$ for $p \in[1, \infty)$ and between $c_{0}\left(c_{0}(E)\right)=$ $c_{0}\left(\mathbb{N}^{2}, E\right)$ and $c_{0}(E)$ (simply due to the fact that $\mathbb{N}$ and $\mathbb{N}^{2}$ have the same cardinality). This simple observation lies at the heart of the proof of our first theorem:

Theorem 2.1. Let E be a Banach space. Then:

(i) for $p \in[1, \infty)$, the Banach algebra $\mathcal{B}\left(\ell^{p}(E)\right)$ is amenable if and only if $\ell^{\infty}\left(\mathcal{B}\left(\ell^{p}(E)\right)\right)$ is amenable;

(ii) $\mathcal{B}\left(c_{0}(E)\right)$ is amenable if and only if $\ell^{\infty}\left(\mathcal{B}\left(c_{0}(E)\right)\right)$ is amenable.

Proof. We only prove (i); (ii) is proven analogously.

Set $\mathfrak{A}:=\ell^{\infty}\left(\mathcal{B}\left(\ell^{p}(E)\right)\right)$. It is elementary that $\mathcal{B}\left(\ell^{p}(E)\right)$ is amenable if $\mathfrak{A}$ is ([Run, Corollary 2.3.2]). 
For the converse, suppose that $\mathcal{B}\left(\ell^{p}(E)\right)$ is amenable, and let $\left(\boldsymbol{d}_{\alpha}\right)_{\alpha \in \mathbb{A}}$ be an approximate diagonal for it; we may suppose that $\Delta \boldsymbol{d}_{\alpha}=\operatorname{id}_{\ell^{p}(E)}$ for all $\alpha \in \mathbb{A}$.

First, observe that we can identify $\mathfrak{A}$ with the block diagonal matrices in $\mathcal{B}\left(\ell^{p}\left(\ell^{p}(E)\right)\right)$. For $n \in \mathbb{N}$, let $P_{n}: \ell^{p}\left(\ell^{p}(E)\right) \rightarrow \ell^{p}(E)$ denote the projection onto the $n$th coordinate. Define

$$
\mathcal{Q}: \mathcal{B}\left(\ell^{p}\left(\ell^{p}(E)\right)\right) \rightarrow \mathcal{B}\left(\ell^{p}\left(\ell^{p}(E)\right)\right), \quad T \mapsto \sum_{n=1}^{\infty} P_{n} T P_{n},
$$

where the infinite series converges in the strong operator topology (SOT). Then $\mathcal{Q}$ is a projection onto $\mathfrak{A}$.

Since we have $\ell^{p}\left(\ell^{p}(E)\right) \cong \ell^{p}(E)$, there are bounded sequences $\left(U_{n}\right)_{n=1}^{\infty}$ and $\left(V_{n}\right)_{n=1}^{\infty}$ in $\mathcal{B}\left(\ell^{p}\left(\ell^{p}(E)\right)\right)$ such that

$$
U_{n} V_{n}=\operatorname{id}_{\ell^{p}\left(\ell^{p}(E)\right)} \quad \text { and } \quad V_{n} U_{n}=P_{n} \quad(n \in \mathbb{N}),
$$

and consequently,

$$
V_{n}=P_{n} V_{n} \quad \text { and } \quad U_{n}=U_{n} P_{n} \quad(n \in \mathbb{N}) .
$$

Define

$$
\begin{aligned}
\mathcal{Q}_{L}: \mathcal{B}\left(\ell^{p}\left(\ell^{p}(E)\right)\right) \rightarrow \mathcal{B}\left(\ell^{p}\left(\ell^{p}(E)\right)\right), & T \mapsto \sum_{n=1}^{\infty} P_{n} T U_{n}, \\
\mathcal{Q}_{R}: \mathcal{B}\left(\ell^{p}\left(\ell^{p}(E)\right)\right) \rightarrow \mathcal{B}\left(\ell^{p}\left(\ell^{p}(E)\right)\right), & T \mapsto \sum_{n=1}^{\infty} V_{n} T P_{n},
\end{aligned}
$$

where again the series are convergent in the strong operator topology. It is obvious that $\mathcal{Q}_{L}$ is a left and $\mathcal{Q}_{R}$ a right $\mathfrak{A}$-module homomorphism, and with (3) in mind, it is easy to see that both $\mathcal{Q}_{L}$ and $\mathcal{Q}_{R}$ attain their values in $\mathfrak{A}$.

Let $S, T \in \mathcal{B}\left(\ell^{p}\left(\ell^{p}(E)\right)\right)$. Since multiplication is jointly continuous on norm bounded subsets with respect to SOT, we have

$$
\begin{aligned}
\left(\mathcal{Q}_{L} S\right)\left(\mathcal{Q}_{R} T\right) & =\text { SOT }-\lim _{N \rightarrow \infty}\left(\sum_{n=1}^{N} P_{n} S U_{n}\right)\left(\sum_{n=1}^{N} V_{n} T P_{n}\right) \\
& =\text { SOT }-\lim _{N \rightarrow \infty} \sum_{n, m=1}^{N} P_{n} S U_{n} V_{m} T P_{m} \\
& =\text { SOT }-\lim _{N \rightarrow \infty} \sum_{n=1}^{N} P_{n} S U_{n} V_{n} T P_{n} \\
& =\text { SOT }-\lim _{N \rightarrow \infty} \sum_{n=1}^{N} P_{n} S T P_{n}=\mathcal{Q}(S T) .
\end{aligned}
$$

It follows that

$$
\Delta \circ\left(\mathcal{Q}_{L} \otimes \mathcal{Q}_{R}\right)=\mathcal{Q} \circ \Delta
$$


Identifying $\mathcal{B}\left(\ell^{p}(E)\right)$ and $\mathcal{B}\left(\ell^{p}\left(\ell^{p}(E)\right)\right.$, we claim that $\left(\left(\mathcal{Q}_{L} \otimes \mathcal{Q}_{R}\right) \boldsymbol{d}_{\alpha}\right)_{\alpha \in \mathbb{A}}$ is an approximate diagonal for $\mathfrak{A}$. Clearly, the net is bounded in $\mathfrak{A} \hat{\otimes} \mathfrak{A}$. Since $\mathcal{Q}_{L} \otimes \mathcal{Q}_{R}$ is an $\mathfrak{A}$-bimodule homomorphism, it follows that (1) holds. Finally, since

$$
\Delta\left(\left(\mathcal{Q}_{L} \otimes \mathcal{Q}_{R}\right) \boldsymbol{d}_{\alpha}\right)=\mathcal{Q}\left(\Delta \boldsymbol{d}_{\alpha}\right)=\mathcal{Q}\left(\operatorname{id}_{\ell^{p}\left(\ell^{p}(E)\right)}\right)=\operatorname{id}_{\ell^{p}\left(\ell^{p}(E)\right)}
$$

by (4), condition (2) holds as well.

Specializing to $E=\mathbb{C}$ yields:

Corollary 2.2. Let $p \in(1, \infty)$ be such that $\mathcal{B}\left(\ell^{p}\right)$ is amenable. Then $\ell^{\infty}\left(\mathcal{B}\left(\ell^{p}\right)\right)$ and $\ell^{\infty}\left(\mathcal{K}\left(\ell^{p}\right)\right)$ are both amenable.

Proof. The claim for $\ell^{\infty}\left(\mathcal{B}\left(\ell^{p}\right)\right)$ is an immediate consequence of Theorem 2.1. Since $\mathcal{K}\left(\ell^{p}\right)$ has a bounded approximate identity, so does $\ell^{\infty}\left(\mathcal{K}\left(\ell^{p}\right)\right)$. Since $\ell^{\infty}\left(\mathcal{K}\left(\ell^{p}\right)\right)$ is a closed ideal of the amenable Banach algebra $\ell^{\infty}\left(\mathcal{B}\left(\ell^{p}\right)\right)$, it is amenable by [Run, Proposition 2.3.3].

REMARKS.

1. The analogous statement of Corollary 2.2 for $\ell^{1}$ and $c_{0}$ is also true. Since, however, $\mathcal{B}\left(\ell^{1}\right)$ and $\mathcal{B}\left(c_{0}\right)$ are known not to be amenable by [Oza], it would be somewhat pointless to formulate it.

2. Even though $\mathcal{B}\left(\ell^{1}\right)$ and $\mathcal{B}\left(c_{0}\right)$ are not amenable, it seems to be unknown whether $\ell^{\infty}\left(\mathcal{K}\left(\ell^{1}\right)\right)$ and $\ell^{\infty}\left(\mathcal{K}\left(c_{0}\right)\right)$ are.

3. It is known that $\ell^{\infty}\left(\mathcal{K}\left(\ell^{2}\right)\right)$ is not amenable (see [L-L-W]), but proving it is at about the same level of difficulty as a proof for the nonamenability of $\mathcal{B}\left(\ell^{2}\right)$.

We shall thus, from now on, focus on the (non-)amenability of $\ell^{\infty}\left(\mathcal{K}\left(\ell^{p}\right)\right)$ instead of that of $\mathcal{B}\left(\ell^{p}\right)$.

3. Ultra-amenability of $\mathcal{K}\left(\ell^{p}\right)$. Let $E$ be a Banach space, let $\mathbb{I}$ be an index set, and let $\mathcal{U}$ be an ultrafilter over $\mathbb{I}$. We let

$$
\mathcal{N}_{\mathcal{U}}:=\left\{\left(x_{i}\right)_{i \in \mathbb{I}}: \lim _{i \in \mathcal{U}}\left\|x_{i}\right\|=0\right\} .
$$

It is immediate that $\mathcal{N}_{\mathcal{U}}$ is a closed subspace of $\ell^{\infty}(\mathbb{I}, E)$. The quotient space $\ell^{\infty}(\mathbb{I}, E) / \mathcal{N}_{\mathcal{U}}$ is called the ultrapower of $E$ with respect to $\mathcal{U}$; we denote it by $(E)_{\mathcal{U}}$. Whenever $\left(x_{i}\right)_{i \in \mathbb{I}} \in \ell^{\infty}(\mathbb{I}, E)$, we write $\left(x_{i}\right)_{\mathcal{U}}$ for its equivalence class in $\mathcal{U}$. For further material on ultrapowers, we refer to the survey article [Hei] and the somewhat more detailed treatment in [Sim].

If $\mathfrak{A}$ is a Banach algebra, then it is straightforward that $(\mathfrak{A})_{\mathcal{U}}$ is again a Banach algebra. The following definition is due to the first author ([Daw 2]):

Definition 3.1. A Banach algebra $\mathfrak{A}$ is said to be ultra-amenable if $(\mathfrak{A})_{\mathcal{U}}$ is amenable for every ultrafilter $\mathcal{U}$. 
REMARK. Ultra-amenability implies amenability ([Daw 2, Corollary 5.5]), but is much stronger: a $C^{*}$-algebra is ultra-amenable if and only if it is subhomogeneous ([Daw 2, Theorem 5.7]), and $\ell^{1}(G)$, for a discrete group $G$, is ultra-amenable if and only if $G$ is finite ([Daw 2, Theorem 5.11]).

Suppose that $\mathcal{B}\left(\ell^{p}\right)$ is amenable for some $p \in(1, \infty)$. Then $\ell^{\infty}\left(\mathcal{K}\left(\ell^{p}\right)\right)$ is amenable by Corollary 2.2, so that its quotient $\left(\mathcal{K}\left(\ell^{p}\right)\right)_{\mathcal{U}}$ is amenable for every ultrafilter $\mathcal{U}$ over $\mathbb{N}$. Alas, this does not allow us (yet) to say that $\mathcal{K}\left(\ell^{p}\right)$ is ultra-amenable because Definition 3.1 requires us to consider ultrafilters over arbitrary index sets.

Nevertheless, the amenability of $\ell^{\infty}\left(\mathcal{K}\left(\ell^{p}\right)\right)$ allows us to conclude the ultra-amenability of $\mathcal{K}\left(\ell^{p}\right)$ by virtue of the following theorem:

THEOREM 3.2. The following are equivalent for a separable Banach algebra $\mathfrak{A}$ :

(i) $\ell^{\infty}(\mathfrak{A})$ is amenable;

(ii) $\ell^{\infty}(\mathbb{I}, \mathfrak{A})$ is amenable for every index set $\mathbb{I}$;

(iii) $\mathfrak{A}$ is ultra-amenable.

For the proof, recall the following definitions from [Daw 2]. Let $\mathfrak{A}$ be a Banach algebra, and let $n \in \mathbb{N}$. Then:

- let $S_{n}(\mathfrak{A})$ denote the collection of all subsets of the unit sphere of $\mathfrak{A}$ of cardinality $n$;

- for $C \geq 1$ and $\varepsilon>0$, let $D_{n}(\mathfrak{A}, C, \varepsilon)$ consist of those $A \subset S_{n}(\mathfrak{A})$ such that there is a sequence $\left(t_{k}\right)_{k=1}^{\infty}$ in $[0, \infty)$ with $\sum_{k=1}^{\infty} t_{k} \leq C$ and with the property that, for each $S \in A$, there are sequences $\left(a_{k}\right)_{k=1}^{\infty}$ and $\left(b_{k}\right)_{k=1}^{\infty}$ in $\mathfrak{A}$ with $\left\|a_{k}\right\|\left\|b_{k}\right\| \leq t_{k}$ for $k \in \mathbb{N}$, so that

and

$$
\boldsymbol{d}:=\sum_{k=1}^{\infty} a_{k} \otimes b_{k} \in \mathfrak{A} \hat{\otimes} \mathfrak{A},
$$

$$
\|a \cdot \boldsymbol{d}-\boldsymbol{d} \cdot a\| \leq \varepsilon \quad \text { and } \quad\left\|\Delta_{\mathfrak{A}}(\boldsymbol{d}) a-a\right\| \leq \varepsilon \quad(a \in F) .
$$

LEMMA 3.3. For a Banach algebra $\mathfrak{A}$ consider the following statements:

(i) there is $C \geq 1$ such that $S_{n}(\mathfrak{A}) \in D_{n}(\mathfrak{A}, C, \varepsilon)$ for each $n \in \mathbb{N}$ and $\varepsilon>0$

(ii) $\ell^{\infty}(\mathbb{I}, \mathfrak{A})$ is amenable for each index set $\mathbb{I}$;

(iii) $\ell^{\infty}(\mathfrak{A})$ is amenable.

Then (i) $\Rightarrow($ ii) $\Rightarrow$ (iii), and (iii) implies (i) if $\mathfrak{A}$ is separable.

Proof. (i) $\Rightarrow$ (ii) is routine in view of the definition of $D_{n}(\mathfrak{A}, C, \varepsilon)$, and (ii) $\Rightarrow$ (iii) is trivial.

Suppose that (iii) holds and that $\mathfrak{A}$ is separable. Let $C \geq 1$ be such that $\mathfrak{A}$ is $C$-amenable, let $n \in \mathbb{N}$, and let $\varepsilon>0$. 
Define a metric $d$ on $S_{n}(\mathfrak{A})$ by letting

$$
d(A, B):=\max _{a \in A} \min _{b \in B}\|a-b\|+\max _{b \in B} \min _{a \in A}\|a-b\| \quad\left(A, B \in S_{n}(\mathfrak{A})\right) .
$$

The separability of $\mathfrak{A}$ implies that the metric space $\left(S_{n}(\mathfrak{A}), d\right)$ is separable and so contains a dense, countable subset, say $\left\{A_{1}, A_{2}, \ldots\right\}$. For each $k \in \mathbb{N}$, let $A_{k}=\left\{a_{1}^{(k)}, \ldots, a_{n}^{(k)}\right\}$. For $j=1, \ldots, n$, set $a_{j}:=\left(a_{j}^{(k)}\right)_{k=1}^{\infty} \in \ell^{\infty}(\mathfrak{A})$. Let $\left(b_{k}\right)_{k=1}^{\infty}$ and $\left(c_{k}\right)_{k=1}^{\infty}$ be sequences in $\ell^{\infty}(\mathfrak{A})$ with $\sum_{k=1}^{\infty}\left\|b_{k}\right\|\left\|c_{k}\right\| \leq C$ such that, for $\boldsymbol{d}:=\sum_{k=1}^{\infty} b_{k} \otimes c_{k} \in \ell^{\infty}(\mathfrak{A}) \hat{\otimes} \ell^{\infty}(\mathfrak{A})$, we have

$$
\left\|a_{j} \cdot \boldsymbol{d}-\boldsymbol{d} \cdot a_{j}\right\| \leq \varepsilon / 2
$$

and

$$
\left\|\Delta_{\ell \infty(\mathfrak{A})}(\boldsymbol{d}) a_{j}-a_{j}\right\| \leq \varepsilon / 2
$$

for $j=1, \ldots, n$. (The existence of such $\left(b_{k}\right)_{k=1}^{\infty}$ and $\left(c_{k}\right)_{k=1}^{\infty}$ follows from the $C$-amenability of $\ell^{\infty}(\mathfrak{A})$.)

For each $k \in \mathbb{N}$, let $b_{k}=\left(b_{\nu}^{(k)}\right)_{\nu=1}^{\infty}$ and $c_{k}=\left(c_{\nu}^{(k)}\right)_{\nu=1}^{\infty}$. Then (6) yields

$$
\sup _{\nu \in \mathbb{N}}\left\|\sum_{k=1}^{\infty} b_{\nu}^{(k)} c_{\nu}^{(k)} a_{j}^{(\nu)}-a_{j}^{(\nu)}\right\| \leq \varepsilon / 2 \quad(j=1, \ldots, n) .
$$

For $\nu \in \mathbb{N}$, let $P_{\nu}: \ell^{\infty}(\mathfrak{A}) \rightarrow \mathfrak{A}$ be the projection onto the $\nu$ th coordinate, and note that $P_{\nu} \otimes P_{\nu}: \ell^{\infty}(\mathfrak{A}) \hat{\otimes} \ell^{\infty}(\mathfrak{A}) \rightarrow \mathfrak{A} \hat{\otimes} \mathfrak{A}$ is a contraction. From (5), we conclude that

(8) $\sup _{\nu \in \mathbb{N}}\left\|\sum_{k=1}^{\infty} a_{j}^{(\nu)} b_{\nu}^{(k)} \otimes c_{\nu}^{(k)}-\sum_{k=1}^{\infty} b_{\nu}^{(k)} \otimes c_{\nu}^{(k)} a_{j}^{(\nu)}\right\| \leq \varepsilon / 2 \quad(j=1, \ldots, n)$.

Finally, it is straightforward that

$$
\sum_{k=1}^{\infty} \sup _{\nu \in \mathbb{N}}\left\|b_{\nu}^{(k)}\right\|\left\|b_{\nu}^{(k)}\right\| \leq \sum_{k=1}^{\infty}\left\|b_{k}\right\|\left\|c_{k}\right\| \leq C .
$$

Let $A \in S_{n}(\mathfrak{A})$ be arbitrary. Since $\left\{A_{1}, A_{2}, \ldots\right\}$ is dense in $S_{n}(\mathfrak{A})$, there is $\nu \in \mathbb{N}$ such that $d\left(A, A_{\nu}\right) \leq \varepsilon / 4 C$. With $A_{\nu}=\left\{a_{1}^{(\nu)}, \ldots, a_{n}^{(\nu)}\right\}$, this means that, for any $a \in A$, there is $j \in\{1, \ldots, n\}$ such that $\left\|a_{j}^{(\nu)}-a\right\| \leq \varepsilon / 4 C$. From (8), we infer that

$$
\begin{aligned}
& \left\|\sum_{k=1}^{\infty} a b_{\nu}^{(k)} \otimes c_{\nu}^{(k)}-\sum_{k=1}^{\infty} b_{\nu}^{(k)} \otimes c_{\nu}^{(k)} a\right\| \\
& \quad \leq 2\left\|a-a_{j}^{(\nu)}\right\| \sum_{k=1}^{\infty}\left\|b_{\nu}^{(k)}\right\|\left\|b_{\nu}^{(k)}\right\|+\left\|\sum_{k=1}^{\infty} a_{j}^{(\nu)} b_{\nu}^{(k)} \otimes c_{\nu}^{(k)}-\sum_{k=1}^{\infty} b_{\nu}^{(k)} \otimes c_{\nu}^{(k)} a_{j}^{(\nu)}\right\| \\
& \quad \leq \frac{\varepsilon}{4 C} \cdot 2 C+\frac{\varepsilon}{2}=\varepsilon
\end{aligned}
$$


and from (7) that

$$
\begin{aligned}
& \left\|\sum_{k=1}^{\infty} b_{\nu}^{(k)} c_{\nu}^{(k)} a-a\right\| \\
& \quad \leq\left\|a-a_{j}^{(\nu)}\right\|+\left\|a-a_{j}^{(\nu)}\right\| \sum_{k=1}^{\infty}\left\|b_{\nu}^{(k)}\right\|\left\|b_{\nu}^{(k)}\right\|+\left\|\sum_{k=1}^{\infty} b_{\nu}^{(k)} c_{\nu}^{(k)} a_{j}^{(\nu)}-a_{j}^{(\nu)}\right\| \\
& \quad \leq \frac{\varepsilon}{4 C}(1+C)+\frac{\varepsilon}{2} \leq \varepsilon .
\end{aligned}
$$

All in all, we have established that $S_{n}(\mathfrak{A}) \in D_{n}(\mathfrak{A}, C, \varepsilon)$.

Proof of Theorem 3.2. By Lemma 3.3, (i) $\Leftrightarrow$ (ii) holds, and (ii) $\Rightarrow$ (iii) is trivial.

We postpone the actual proof of (iii) $\Rightarrow$ (i) for some preliminary considerations.

Let $\mathcal{S}$ be the set of all sequences in $[0, \infty)$. For $\left(t_{k}\right)_{k=1}^{\infty},\left(s_{k}\right)_{k=1}^{\infty} \in \mathcal{S}$, define $\left(t_{k}\right)_{k=1}^{\infty} \ll\left(s_{k}\right)_{k=1}^{\infty}$ if there are $\left(r_{k}\right)_{k=1}^{\infty} \in \mathcal{S}$, a bijection $\sigma: \mathbb{N} \rightarrow \mathbb{N}$, and $\nu, \mu \in \mathbb{N}$ such that

- $s_{k}=r_{\sigma(k)}$ for $k \in \mathbb{N}$,

- $r_{k}=t_{k}$ for $k<\nu$,

- $r_{\nu}+r_{\nu+1}+\cdots+r_{\nu+\mu-1}=t_{\nu}$, and

- $r_{k+\mu-1}=t_{k}$ for $k>\nu$.

(Informally, one might want to say that $\left(r_{k}\right)_{k=1}^{\infty}$ is obtained from $\left(t_{k}\right)_{k=1}^{\infty}$ by splitting up one term and from $\left(s_{k}\right)_{k=1}^{\infty}$ through rearrangement.) It is clear from this definition that, whenever $\left(t_{k}\right)_{k=1}^{\infty} \ll\left(s_{k}\right)_{k=1}^{\infty}$ and one of the sequences lies in $\ell^{1}$, then so does the other and has the same norm in $\ell^{1}$. We then define $\left(t_{k}\right)_{k=1}^{\infty} \preceq\left(s_{k}\right)_{k=1}^{\infty}$ if there are $\left(r_{k}^{(1)}\right)_{k=1}^{\infty}, \ldots,\left(r_{k}^{(m)}\right)_{k=1}^{\infty} \in \mathcal{S}$ such that

$$
\left(t_{k}\right)_{k=1}^{\infty} \ll\left(r_{k}^{(1)}\right)_{k=1}^{\infty} \ll \cdots \ll\left(r_{k}^{(m)}\right)_{k=1}^{\infty} \ll\left(s_{k}\right)_{k=1}^{\infty} .
$$

Let $\mathcal{S}_{0}$ be the collection of all sequences in $\mathcal{S}$ that are eventually zero, and note that $\left(\mathcal{S}_{0}, \preceq\right)$ is a directed set.

Let $n \in \mathbb{N}$, let $F \in S_{n}(\mathfrak{A})$, let $\varepsilon>0$, and let $\left(t_{k}\right)_{k=1}^{\infty} \in \ell^{1} \cap S$. We call $F$ and $\left(t_{k}\right)_{k=1}^{\infty}$ compatible if there is $\boldsymbol{d}=\sum_{k=1}^{\infty} a_{k} \otimes b_{k} \in \mathfrak{A} \hat{\otimes} \mathfrak{A}$ with $\left\|a_{k}\right\|\left\|b_{k}\right\| \leq t_{k}$ for $k \in \mathbb{N}$ and

$$
\|a \cdot \boldsymbol{d}-\boldsymbol{d} \cdot a\| \leq \varepsilon \quad \text { and } \quad\|\Delta(\boldsymbol{d}) a-a\| \leq \varepsilon \quad(a \in F) .
$$

Note that, if $\left(t_{k}\right)_{k=1}^{\infty} \preceq\left(s_{k}\right)_{k=1}^{\infty}$ and $F$ and $\left(t_{k}\right)_{k=1}^{\infty}$ are compatible, then so are $F$ and $\left(s_{k}\right)_{k=1}^{\infty}$.

Suppose now that $\mathfrak{A}$ is ultra-amenable, let $C \geq 1$ be as in [Daw 2, Theorem 5.6], let $n \in \mathbb{N}$, and let $\varepsilon>0$. By [Daw 2, Theorem 5.6], there is a partition of $S_{n}(\mathfrak{A})$ into finitely many sets each of which has a compatible sequence in $\ell^{1} \cap \mathcal{S}$ with $\ell^{1}$-norm at most $C$. We can suppose that each of 
these sequences lies in $\mathcal{S}_{0}$ and use the directedness of $\left(\mathcal{S}_{0}, \preceq\right)$ to obtain one single sequence in $\mathcal{S}_{0}$ - still with $\ell^{1}$-norm at most $C$ - that is compatible with all sets in the partition of $S_{n}(\mathfrak{A})$. But then $S_{n}(\mathfrak{A})$ itself and that sequence are compatible, which means that $S_{n}(\mathfrak{A}) \in D_{n}(\mathfrak{A}, C, \varepsilon)$. By Lemma 3.3, this implies the amenability of $\ell^{\infty}(\mathfrak{A})$.

REMARK. The proof of Theorem 3.2 can be modified to yield the following generalization: a Banach algebra $\mathfrak{A}$ of density character $\kappa$ is ultraamenable if and only if $\ell^{\infty}(\mathbb{I}, \mathfrak{A})$ is amenable for any index set $\mathbb{I}$ and if and only if it is amenable for an index set $\mathbb{I}$ of cardinality $\kappa$. This closes a gap in the proof of [L-L-W, Theorem 2.5], which claims the equivalence of Theorem 3.2 (ii) and (iii) in the case of a $C^{*}$-algebra.

As $\mathcal{K}\left(\ell^{p}\right)$ for $p \in(1, \infty)$ and $\mathcal{K}\left(c_{0}\right)$ are separable, Theorem 3.2 yields:

Corollary 3.4. Let $E=\ell^{p}$ with $p \in(1, \infty)$ or $E=c_{0}$. Then the following are equivalent:

(i) $\ell^{\infty}(\mathcal{K}(E))$ is amenable;

(ii) $\ell^{\infty}(\mathbb{I}, \mathcal{K}(E))$ is amenable for every index set $\mathbb{I}$;

(iii) $\mathcal{K}(E)$ is ultra-amenable.

4. Amenability of $\ell^{\infty}(\mathcal{K}(E))$ for $\mathcal{L}^{p}$-spaces. Whether or not the Banach algebras of the form $\ell^{\infty}(\mathcal{K}(E))$ for a Banach space $E$ are amenable seems to have received very little attention in the literature so far. As Corollary 2.2 shows, it is, for $E=\ell^{p}$ with $p \in[1, \infty)$, intimately linked to the open problem of whether $\mathcal{B}\left(\ell^{p}\right)$ is amenable and thus certainly a question deserving further exploration.

In this section, we show that the (possible) amenability of $\ell^{\infty}\left(\mathcal{K}\left(\ell^{p}\right)\right)$ entails the amenability of $\ell^{\infty}(\mathcal{K}(E))$ for a large class of Banach spaces $E$.

For our first proposition, we denote by $\mathcal{F}(E, F)$, for two Banach spaces $E$ and $F$, the bounded finite rank operators from $E$ to $F$; as usual, we write $\mathcal{F}(E)$ as shorthand for $\mathcal{F}(E, E)$.

Proposition 4.1. Let $E$ and $F$ be Banach spaces such that $E^{*}$ and $F^{*}$ have the bounded approximation property. Suppose further that the following factorization property holds:

there is $C \geq 0$ such that, for each $T \in \mathcal{F}(F)$, there are $S \in \mathcal{F}(F, E)$ and $R \in \mathcal{F}(E, F)$ with $\|R\|\|S\| \leq C\|T\|$ and $R S=T$.

Then, for any index set $\mathbb{I}$, the following are equivalent:

(i) $\ell^{\infty}(\mathbb{I}, \mathcal{K}(E))$ is amenable;

(ii) $\ell^{\infty}(\mathbb{I}, \mathcal{K}(E \oplus F))$ is amenable.

Proof. Let $\mathbb{I}$ be an index set, and let $\mathfrak{A}:=\ell^{\infty}(\mathbb{I}, \mathcal{K}(E \oplus F))$. We wish to apply Theorem 1.2. 
As both $E^{*}$ and $F^{*}$ have the bounded approximation property, so does $(E \oplus F)^{*} \cong E^{*} \oplus F^{*}$. Consequently, $\mathcal{K}(E \oplus F)$ has a bounded approximate identity, and so does $\mathfrak{A}$.

For $i \in \mathbb{I}$, let $P_{1, i}: E \oplus F \rightarrow E$ and $P_{2, i}: E \oplus F \rightarrow F$ be the canonical projections; for $j=1,2$, set $P_{j}:=\left(P_{j, i}\right)_{i \in \mathbb{I}} \in \ell^{\infty}(\mathbb{I}, \mathcal{B}(E \oplus F)) \subset$ $\mathcal{M}\left(\ell^{\infty}(\mathbb{I}, \mathcal{K}(E \oplus F))\right)$. It follows that $P_{1} \mathfrak{A} P_{1} \cong \ell^{\infty}(\mathbb{I}, \mathcal{K}(E))$ and $P_{2} \mathfrak{A} P_{2} \cong$ $\ell^{\infty}(\mathbb{I}, \mathcal{K}(F))$.

The restriction of $\Delta_{\mathfrak{A}}$ to $P_{2} \mathfrak{A} P_{1} \hat{\otimes} P_{1} \mathfrak{A} P_{2}$ induces a quotient norm, say $|\cdot|$, on its range, which dominates the given norm $\|\cdot\|$. By our factorization hypothesis, the range of $\Delta_{\mathfrak{A}}\left(P_{2} \mathfrak{A} P_{1} \hat{\otimes} P_{1} \mathfrak{A} P_{2}\right)$ contains $\ell^{\infty}(\mathcal{F}(F))$, and we have $|\cdot| \leq C\|\cdot\|$ on $\ell^{\infty}(\mathcal{F}(F))$. As $F^{*}$ has the approximation property, so does $F$, and in particular, $\mathcal{F}(F)$ is dense in $\mathcal{K}(F)$, as is $\ell^{\infty}(\mathcal{F}(F))$ in $\ell^{\infty}(\mathcal{K}(F))$. Every element of $\ell^{\infty}(\mathcal{K}(F))$ is thus a limit - with respect to $\|\cdot\|$ of a sequence in $\ell^{\infty}(\mathcal{F}(F))$. It is a Cauchy sequence with respect to $\|\cdot\|$ and thus with respect to $|\cdot|$; consequently, it converges - with respect to $|\cdot|$ - to an element in $\Delta_{\mathfrak{A}}\left(P_{2} \mathfrak{A} P_{1} \hat{\otimes} P_{1} \mathfrak{A} P_{2}\right)$. Since $\|\cdot\| \leq|\cdot|$, this limit with respect to $|\cdot|$ is the same limit as with respect to $\|\cdot\|$. So, $\Delta_{\mathfrak{A}}$ maps $P_{2} \mathfrak{A} P_{1} \hat{\otimes} P_{1} \mathfrak{A} P_{2}$ onto $P_{2} \mathfrak{A} P_{2}$.

The hypotheses of Theorem 1.2 are thus all satisfied, and the claim follows.

We shall now look at Banach spaces for which the factorization hypothesis of Proposition 4.1 is satisfied.

Let $p \in[1, \infty]$ and $\lambda \geq 1$. A Banach space $E$ is called an $\mathcal{L}_{\lambda}^{p}$-space if, for any finite-dimensional subspace $X$ of $E$, there are $n \in \mathbb{N}$ and an $n$-dimensional subspace $Y$ of $E$ containing $X$ such that $d\left(Y, \ell_{n}^{p}\right) \leq \lambda$, where $d$ is the Banach-Mazur distance ([L-P, Definition 3.1]). We call $E$ simply an $\mathcal{L}^{p}$-space if it is an $\mathcal{L}_{\lambda}^{p}$-space for some $\lambda \geq 1$. All $L^{p}$-spaces, i.e., spaces of $p$-integrable functions on some measure space, are $\mathcal{L}^{p}$-spaces. The $\mathcal{L}^{p}$-spaces were introduced in $[\mathrm{L}-\mathrm{P}]$ and studied further in $[\mathrm{L}-\mathrm{R}]$. We list some of their properties:

- Every $\mathcal{L}^{p}$-space is isomorphic to a subspace of an $L^{p}$-space $([\mathrm{L}-\mathrm{R}$, Theorem I(i)]). In particular, for $p \in(1, \infty)$, each $\mathcal{L}^{p}$-space is reflexive.

- If $E$ is an $\mathcal{L}^{p}$-space, then $E^{*}$ is an $\mathcal{L}^{p^{\prime}}$-space, where $p^{\prime} \in[1, \infty]$ is conjugate to $p$, i.e., satisfies $1 / p+1 / p^{\prime}=1$ ([L-R, Theorem III(a)]).

- If $E$ is an $\mathcal{L}^{p}$-space, then there is a constant $\varrho \geq 1$ such that, for each finite-dimensional subspace $X$ of $E$, there are $n \in \mathbb{N}$, an $n$-dimensional subspace $Y$ of $E$ containing $X$ with $d\left(Y, \ell_{n}^{p}\right)$, and a projection $P$ onto $Y$ with $\|P\| \leq \varrho([\mathrm{L}-\mathrm{R}$, Theorem $\operatorname{III}(\mathrm{c})])$. In particular, $E$ has the bounded approximation property.

In [G-J-W], it is mentioned without proof before [G-J-W, Theorem 6.4] that, given any two infinite-dimensional $\mathcal{L}^{p}$-spaces $E$ and $F$, every operator 
in $\mathcal{F}(F)$ factors through $E$ with both factors being compact. Since, for our purpose, we need control over the norms of those factors, we give a refinement of this observation with a detailed proof:

Lemma 4.2. Let $p \in[1, \infty]$, and let $E$ and $F$ be $\mathcal{L}^{p}$-spaces with $\operatorname{dim} E=\infty$. Then there is $C \geq 0$ such that, for each $T \in \mathcal{F}(F)$, there are $S \in \mathcal{F}(F, E)$ and $R \in \mathcal{F}(E, F)$ with $\|R\|\|S\| \leq C\|T\|$ and $R S=T$.

Proof. Let $T \in \mathcal{F}(F)$, and set $X:=T F$. Let $\lambda \geq 1$ be such that $E$ is an $\mathcal{L}_{\lambda}^{p}$-space. Then there are $n \in \mathbb{N}$, a finite-dimensional subspace $Y$ of $F$ containing $X$, and a bijective linear map $\tau: Y \rightarrow \ell_{n}^{p}$ such that $\|\tau\|\left\|\tau^{-1}\right\| \leq \lambda$.

Let $\varrho \geq 1$ be the constant for $E$ whose existence is guaranteed by [L-R, Theorem III(c)]. Let $Z_{0}$ be an $n$-dimensional subspace of $E$. (Here, we require that $\operatorname{dim} E=\infty$.) Then there are $m \in \mathbb{N}$, an $m$-dimensional subspace $Z$ of $E$ containing $Z_{0}$, a bijective map $\sigma: Z \rightarrow \ell_{m}^{p}$ with $\|\sigma\|\left\|\sigma^{-1}\right\| \leq \varrho$, and a projection $P$ onto $Z$ with $\|P\| \leq \varrho$; note that necessarily $m \geq n$. With $\iota: \ell_{n}^{p} \rightarrow \ell_{m}^{p}$ and $\pi: \ell_{m}^{p} \rightarrow \ell_{n}^{p}$ being the canonical embedding and projection, we see that $T=\tau^{-1} \pi \sigma \sigma^{-1} \iota \tau T$. Set $S:=\sigma^{-1} \iota \tau T$ and $R:=\tau^{-1} \pi \sigma P$. Then $S=R T$, and we have

$$
\begin{aligned}
\|R\|\|S\| & \leq\left\|\tau^{-1}\right\|\|\pi\|\|\sigma\|\|P\|\left\|\sigma^{-1}\right\|\|\iota\|\|\tau\|\|T\| \\
& =\|\tau\|\left\|\tau^{-1}\right\|\|\sigma\|\left\|\sigma^{-1}\right\|\|P\|\|T\| \leq \lambda \varrho^{2}\|T\| .
\end{aligned}
$$

Hence, $C:=\lambda \varrho^{2}$ has the desired property.

Remark. In [D-F], A. Defant and K. Floret introduced the class of $\mathcal{L}_{g^{-}}^{p}$ spaces, which contains all $\mathcal{L}^{p}$-spaces, but is somewhat better behaved. For $p=1, \infty$, the $\mathcal{L}_{g}^{p}$-spaces are just the $\mathcal{L}^{p}$-spaces whereas, for $p \in(1, \infty)$, a space is an $\mathcal{L}_{g}^{p}$-space if and only if it is an $\mathcal{L}^{p}$-space or isomorphic to a Hilbert space ([D-F, 23.3]). Therefore, Lemma 4.2 does not hold true for $\mathcal{L}_{g}^{p}$-spaces if $p \neq 1, \infty$.

Together, Proposition 4.1 and Lemma 4.2 yield the following dichotomy theorem:

TheOREm 4.3. Let $p \in[1, \infty]$, and let $\mathbb{I}$ be an index set. Then one of the following assertions is true:

(i) $\ell^{\infty}(\mathbb{I}, \mathcal{K}(E))$ is amenable for every infinite-dimensional $\mathcal{L}^{p}$-space $E$;

(ii) $\ell^{\infty}(\mathbb{I}, \mathcal{K}(E))$ is not amenable for any infinite-dimensional $\mathcal{L}^{p}$-space $E$.

Proof. Suppose that (ii) is false, i.e., there is an infinite-dimensional $\mathcal{L}^{p}$ space $E$ such that $\ell^{\infty}(\mathbb{I}, \mathcal{K}(E))$ is amenable. Let $F$ be any infinite-dimensional $\mathcal{L}^{p}$-space. Then Lemma 4.2 and Proposition 4.1 imply that $\ell^{\infty}(\mathbb{I}, \mathcal{K}(E \oplus F))$ is amenable. Interchanging the rôles of $E$ and $F$, and invoking Lemma 4.2 and Proposition 4.1 again, then yields the amenability of $\ell^{\infty}(\mathbb{I}, \mathcal{K}(F))$. This proves that (i) is true. 
Combining Theorems 3.2 and 4.3, we obtain:

Corollary 4.4. Let $p \in(1, \infty]$, let $E=\ell^{p}$ if $p \in(1, \infty)$ and $E=c_{0}$ if $p=\infty$, and suppose that $\ell^{\infty}(\mathcal{K}(E))$ is amenable. Then $\ell^{\infty}(\mathbb{I}, \mathcal{K}(F))$ is amenable for every index set $\mathbb{I}$ and every infinite-dimensional $\mathcal{L}^{p}$-space $F$. In particular, $\mathcal{K}(F)$ is ultra-amenable for every infinite-dimensional $\mathcal{L}^{p}$ space $F$.

Remark. Let $p \in(1, \infty) \backslash\{2\}$. Then $\ell^{p}\left(\ell^{2}\right)$ and $\ell^{p} \oplus \ell^{2}$ are not $L^{p}$-spaces, but still $\mathcal{L}^{p}$-spaces ([L-P, Example 8.2]). Hence, if $\ell^{\infty}\left(\mathcal{K}\left(\ell^{p}\right)\right)$ is amenable, then so are $\ell^{\infty}\left(\mathcal{K}\left(\ell^{p}\left(\ell^{2}\right)\right)\right)$ and $\ell^{\infty}\left(\mathcal{K}\left(\ell^{p} \oplus \ell^{2}\right)\right)$. This is remarkable because $\mathcal{B}\left(\ell^{p} \oplus \ell^{2}\right)$ is known not to be amenable ([Grø, Question 4$\left.]\right)$.

5. A non-amenability criterion for $\ell^{\infty}(\mathcal{K}(E \oplus F))$. As we just observed, $\ell^{\infty}\left(\mathcal{K}\left(\ell^{p}\right)\right)$ being amenable implies the amenability of $\ell^{\infty}\left(\mathcal{K}\left(\ell^{p} \oplus \ell^{2}\right)\right)$. In this section, we shall thus explore the amenability of $\ell^{\infty}(\mathcal{K}(E \oplus F))$ for two Banach spaces $E$ and $F$.

Recall that an operator ideal $\mathcal{A}$ is a rule that assigns to each pair $(E, F)$ of Banach spaces a subspace $\mathcal{A}(E, F)$ of $\mathcal{B}(E, F)$ containing $\mathcal{F}(E, F)$ such that $R T S \in \mathcal{A}(X, Y)$ for any Banach spaces $X$ and $Y$ and any $T \in \mathcal{A}(E, F)$, $S \in \mathcal{B}(X, E)$, and $R \in \mathcal{B}(F, Y)$ ); if $E=F$, we convene again to simply write $\mathcal{A}(E)$. The seminal reference on operator ideals is [Pie]. More recent treatments can be found in $[\mathrm{D}-\mathrm{F}],[\mathrm{D}-\mathrm{J}-\mathrm{T}]$, or [T-J]. Following [D-J-T], we call $[\mathcal{A}, \alpha]$ a Banach operator ideal if, for each pair $(E, F)$ of Banach spaces, there is a norm $\alpha$ on $\mathcal{A}(E, F)$ turning it into a Banach space such that

$$
\alpha(y \odot \phi)=\|y\|\|\phi\| \quad\left(y \in F, \phi \in E^{*}\right),
$$

where $y \odot \phi \in \mathcal{F}(E, F)$ is the rank one operator corresponding to the elementary tensor $y \otimes \phi$, and

$$
\alpha(R T S) \leq\|R\| \alpha(T)\|S\| \quad(T \in \mathcal{A}(E, F), S \in \mathcal{B}(X, E), R \in \mathcal{B}(F, Y))
$$

for any Banach spaces $X$ and $Y$. Given a Banach operator ideal $[\mathcal{A}, \alpha]$, its maximal hull $\left[\mathcal{A}^{\max }, \alpha^{\max }\right]$ is defined as follows. For two Banach spaces $E$ and $F$, let $\mathfrak{F}(E)$ denote the finite-dimensional subspaces of $E$, and let $\mathfrak{F}_{\mathrm{c}}(F)$ stand for the closed subspaces of $F$ with finite co-dimension; for $X \in \mathfrak{F}(E)$ and $Y \in \mathfrak{F}_{\mathrm{c}}(F)$, let $\iota_{X}: X \rightarrow E$ and $\pi_{Y}: F \rightarrow F / Y$ be the inclusion and quotient map, respectively. We define

$$
\begin{aligned}
\alpha^{\max }(T):=\sup \left\{\alpha\left(\pi_{Y} T \iota_{X}\right): X \in \mathfrak{F}(E), Y \in \mathfrak{F}_{\mathrm{c}}(F)\right\} & \in[0, \infty] \\
& (T \in \mathcal{B}(E, F))
\end{aligned}
$$

and

$$
\mathcal{A}^{\max }(E, F):=\left\{T \in \mathcal{B}(E, F): \alpha^{\max }(T)<\infty\right\} .
$$

It is routinely checked that $\left[\mathcal{A}^{\max }, \alpha^{\max }\right]$ is again a Banach operator ideal, and we call $[\mathcal{A}, \alpha]$ maximal if $\left[\mathcal{A}^{\max }, \alpha^{\max }\right]=[\mathcal{A}, \alpha]$. 
An immediate consequence of [D-F, 17.5, Representation Theorem for Maximal Operator Ideals] is: if $[\mathcal{A}, \alpha]$ is a maximal Banach operator ideal, then $\mathcal{A}\left(E, F^{*}\right)$ is a dual Banach space for any two Banach spaces $E$ and $F$ such that the weak* topology of $\mathcal{A}\left(E, F^{*}\right)$ coincides with the weak* topology of $\mathcal{B}\left(E, F^{*}\right)=(E \hat{\otimes} F)^{*}$ on norm bounded subsets.

In particular, we have (cf. [D-F, 17.21, Proposition]):

Lemma 5.1. Let $[\mathcal{A}, \alpha]$ be a maximal Banach operator ideal, let $E$ and $F$ be Banach spaces, and let $\left(T_{i}\right)_{i \in \mathbb{I}}$ be a bounded net in $\mathcal{A}\left(E, F^{*}\right)$ that converges to $T \in \mathcal{B}\left(E, F^{*}\right)$ with respect to the weak $k^{*}$ topology of $\mathcal{B}\left(E, F^{*}\right)$. Then $T$ lies in $\mathcal{A}\left(E, F^{*}\right)$.

Proposition 5.2. Let $E$ and $F$ be Banach spaces, let $\mathbb{I}$ be an index set, and suppose that there are $\left(T_{i}\right)_{i \in \mathbb{I}} \in \ell^{\infty}(\mathbb{I}, \mathcal{K}(F, E))$ and an ultrafilter $\mathcal{U}$ over $\mathbb{I}$ such that weak ${ }^{*}-\lim _{i \in \mathcal{U}} T_{i} \notin \mathcal{K}\left(F, E^{* *}\right)$, where the limit is with respect to the weak $k^{*}$ topology of $\mathcal{B}\left(E, F^{* *}\right)$. Suppose further that there is a maximal operator ideal $[\mathcal{A}, \alpha]$ with the following properties:

(a) $\mathcal{K}(E, F) \subset \mathcal{A}(E, F)$ such that the inclusion is continuous;

(b) $\mathcal{A}\left(F, E^{* *}\right) \subset \mathcal{K}\left(F, E^{* *}\right)$.

Then $\ell^{\infty}(\mathbb{I}, \mathcal{K}(E \oplus F))$ is not amenable.

Proof. Let $P_{1}$ and $P_{2}$ be the projections in $\ell^{\infty}(\mathbb{I}, \mathcal{B}(E \oplus F))$ induced by the canonical projections onto $E$ and $F$, respectively (compare the proof of Proposition 4.1). We wish to apply Proposition 1.3.

Letting

$$
I:=\overline{\ell^{\infty}(\mathbb{I}, \mathcal{A}(E \oplus F)) \cap \ell^{\infty}(\mathbb{I}, \mathcal{K}(E \oplus F))},
$$

with the closure taken in the norm topology of $\ell^{\infty}(\mathbb{I}, \mathcal{K}(E \oplus F))$, defines a closed ideal of $\mathfrak{A}:=\ell^{\infty}(\mathbb{I}, \mathcal{K}(E \oplus F))$.

From (a), it is obvious that $P_{2} I P_{1}=P_{2} \mathfrak{A} P_{1}$.

To see that $P_{1} I P_{2} \subsetneq P_{1} \mathfrak{A} P_{2}$, let $\left(T_{i}\right)_{i \in \mathbb{I}} \in \ell^{\infty}(\mathbb{I}, \mathcal{K}(F, E))$ and an ultrafilter $\mathcal{U}$ over $\mathbb{I}$ be as specified in the hypotheses. We claim that $\left(T_{i}\right)_{i \in \mathbb{I}} \in$ $P_{1} \mathfrak{A} P_{2} \backslash P_{1} I P_{2}$. Define

$$
Q_{\mathcal{U}}: \ell^{\infty}(\mathbb{I}, \mathcal{K}(F, E)) \rightarrow \mathcal{B}(F, E), \quad\left(S_{i}\right)_{i \in \mathbb{I}} \mapsto \text { weak }^{*}-\lim _{i \in \mathcal{U}} S_{i},
$$

so that $T:=Q_{\mathcal{U}}\left(\left(T_{i}\right)_{i \in \mathbb{I}}\right) \notin \mathcal{K}\left(F, E^{* *}\right)$. Assume that $\left(T_{i}\right)_{i \in \mathbb{I}} \in P_{1} I P_{2}$, and let $\varepsilon>0$ be arbitrary. By the definition of $I$, there is thus $\left(R_{i}\right)_{i \in \mathbb{I}} \in$ $\ell^{\infty}(\mathbb{I}, \mathcal{A}(F, E)) \cap \ell^{\infty}(\mathbb{I}, \mathcal{K}(F, E))$ such that $\sup _{i \in \mathbb{I}}\left\|R_{i}-T_{i}\right\|<\varepsilon$. Since $Q_{\mathcal{U}}$ is a contraction, this means that $\|R-T\|<\varepsilon$, where $R:=Q_{\mathcal{U}}\left(\left(R_{i}\right)_{i \in \mathbb{I}}\right)$. By Lemma $5.1, R \in \mathcal{A}\left(F, E^{* *}\right)$ holds, so that $R \in \mathcal{K}\left(F, E^{* *}\right)$ by (b). Since $\varepsilon>0$ was arbitrary, this means that $T \in \mathcal{K}\left(F, E^{* *}\right)$, which contradicts our hypotheses. 
The hypotheses of Proposition 5.2 appear to be technical and somewhat contrived, but as our next theorem shows, they do, in fact, occur naturally in certain situations:

TheOREM 5.3. The Banach algebra $\ell^{\infty}\left(\mathcal{K}\left(E \oplus \ell^{2}\right)\right)$ is not amenable for any of the following spaces $E$ : $c_{0}, \ell^{\infty}$, and $\ell^{1}$.

Proof. We first consider the case $E=c_{0}$.

For $n \in \mathbb{N}$, let $\pi_{n}: c_{0} \rightarrow c_{0}$ denote the projection onto the first $n$ coordinates, and let $\iota: \ell^{2} \rightarrow c_{0} \hookrightarrow \ell^{\infty}$ be the natural inclusion. Then $\left(\pi_{n} \iota\right)_{n=1}^{\infty} \in \ell^{\infty}\left(\mathcal{K}\left(\ell^{2}, c_{0}\right)\right)$, and weak ${ }^{*} \lim _{n \in \mathcal{U}} \pi_{n} \iota=\iota \notin \mathcal{K}\left(\ell^{2}, \ell^{\infty}\right)$ for any free ultrafilter $\mathcal{U}$ over $\mathbb{N}$.

Let $\left[\Pi_{2}, \pi_{2}\right]$ be the ideal of 2-summing operators (see [D-J-T, p. 31], for instance); then $\left[\Pi_{2}, \pi_{2}\right]$ is maximal (by [D-J-T, Theorem 6.16]). By [D-J-T, Theorem 3.7] we have $\Pi_{2}\left(c_{0}, \ell^{2}\right)=\mathcal{B}\left(c_{0}, \ell^{2}\right)$, so that Proposition 5.2(a) is satisfied. On the other hand, every 2 -summing operator is completely continuous ([D-J-T, Theorem 2.17]). Since $\ell^{2}$ is reflexive, this means that $\Pi_{2}\left(\ell^{2}, \ell^{\infty}\right) \subset \mathcal{K}\left(\ell^{2}, \ell^{\infty}\right)$, so that Proposition 5.2(b) holds as well.

The $E=\ell^{\infty}$ case has an almost identical proof.

Suppose now that $E=\ell^{1}$. We shall apply Proposition 5.2 to the Banach algebra $\ell^{\infty}\left(\mathcal{K}\left(\ell^{2} \oplus \ell^{1}\right)\right)$, which is isomorphic to $\ell^{\infty}\left(\mathcal{K}\left(\ell^{1} \oplus \ell^{2}\right)\right)$. With $\iota: \ell^{1} \rightarrow$ $\ell^{2}$ being the canonical inclusion and $\pi_{n}: \ell^{2} \rightarrow \ell^{2}$ for $n \in \mathbb{N}$ denoting the projection onto the first $n$ coordinates, we have weak ${ }^{*}-\lim _{n \in \mathcal{U}} \pi_{n} \iota=\iota \notin$ $\mathcal{K}\left(\ell^{1}, \ell^{2}\right)$ for any free ultrafilter $\mathcal{U}$ over $\mathbb{N}$-just as in the case $E=c_{0}$. Let $\left[\Pi_{2}^{d}, \pi_{2}^{d}\right]$ be the dual ideal of $\left[\Pi_{2}, \pi_{2}\right]$ (see [D-J-T, p. 186]), so that $\left[\Pi_{2}^{d}, \pi_{2}^{d}\right]$ is maximal by [D-J-T, Corollary 9.4]. Using the definition of $\left[\Pi_{2}^{d}, \pi_{2}^{d}\right]$ and arguing as in the $E=c_{0}$ case, we see that Proposition 5.2(a) and (b) are satisfied.

REMARK. Even though $\ell^{\infty}\left(\mathcal{K}\left(\ell^{2}\right)\right)$ is known not to be amenable, there seems to be no way-by means of Theorem 1.2, for instance - to conclude directly from its non-amenability that the Banach algebras considered in Theorem 5.3 are not amenable.

Having established the non-amenability of $\ell^{\infty}\left(\mathcal{K}\left(\ell^{p} \oplus \ell^{2}\right)\right)$ for $p=1, \infty$ with the help of Proposition 5.2, one might be tempted to try to extend this result to general $p \in[1, \infty] \backslash\{2\}$ through the choice of a suitable maximal Banach operator ideal $[\mathcal{A}, \alpha]$. Alas, as we shall see now, this attempt is futile:

Proposition 5.4. Let $p \in(1, \infty) \backslash\{2\}$, let $\mathfrak{A}:=\ell^{\infty}\left(\mathcal{K}\left(\ell^{p} \oplus \ell^{2}\right)\right)$, and let $P_{1}, P_{2} \in \ell^{\infty}\left(\mathcal{B}\left(\ell^{p} \oplus \ell^{2}\right)\right)$ be the projections induced by the canonical projections onto $\ell^{p}$ and $\ell^{2}$, respectively. Then there is no closed ideal I of $\mathfrak{A}$ with $P_{2} I P_{1}=P_{2} \mathfrak{A} P_{1}$ and $P_{1} I P_{2} \subsetneq P_{1} \mathfrak{A} P_{2}$. 
Proof. We use the fact ([L-T, p. 73]) that we have an isomorphism

$$
\ell^{p} \cong \ell^{p}-\bigoplus_{n=1}^{\infty} \ell_{n}^{2}
$$

For $n \in \mathbb{N}$, let $J_{n}: \ell_{n}^{2} \rightarrow \ell^{p}$ and $Q_{n}: \ell^{p} \rightarrow \ell_{n}^{2}$ be the embedding of and projection onto the $n$th summand in (9), respectively; note that the maps $J_{n}$ and $P_{n}$ are uniformly bounded. Furthermore, let $\iota_{n}: \ell_{n}^{2} \rightarrow \ell^{2}$ and $\pi_{n}: \ell^{2} \rightarrow \ell_{n}^{2}$ be the canonical embedding and projection, respectively, for $n \in \mathbb{N}$.

Assume that there is a closed ideal $I$ of $\mathfrak{A}$ with $P_{2} I P_{1}=P_{2} \mathfrak{A} P_{1}$. Note that, since $\mathfrak{A}$ has a bounded approximate identity, $I$ is also a closed ideal of $\ell^{\infty}\left(\mathcal{B}\left(\ell^{p} \oplus \ell^{2}\right)\right)$, so that, in particular, $P_{j} I P_{k} \subset I$ for $j, k=1,2$.

Let $\left(T_{n}\right)_{n=1}^{\infty} \in \ell^{\infty}\left(\mathcal{K}\left(\ell^{2}, \ell^{p}\right)\right)$, and let $\varepsilon>0$ be arbitrary. For each $n \in \mathbb{N}$, we can find $S_{n} \in \mathcal{F}\left(\ell^{2}, \ell^{p}\right)$ with $\left\|T_{n}-S_{n}\right\| \leq \varepsilon$ as well as $N_{n} \in \mathbb{N}$ such that $S_{n} \iota_{N_{n}} \pi_{N_{n}}=S_{n}$. Define $\left(U_{n}\right)_{n=1}^{\infty} \in \ell^{\infty}\left(\mathcal{K}\left(\ell^{2}, \ell^{p}\right)\right)$ and $\left(V_{n}\right)_{n=1}^{\infty} \in \ell^{\infty}\left(\mathcal{K}\left(\ell^{p}\right)\right)$ by letting

$$
U_{n}:=\iota_{N_{n}} Q_{N_{n}} \quad \text { and } \quad V_{n}:=J_{N_{n}} \pi_{N_{n}} \quad(n \in \mathbb{N}),
$$

so that

$$
\left[\begin{array}{cc}
0 & \left(S_{n}\right)_{n=1}^{\infty} \\
0 & 0
\end{array}\right]=\left[\begin{array}{cc}
0 & \left(S_{n}\right)_{n=1}^{\infty} \\
0 & 0
\end{array}\right]\left[\begin{array}{cc}
0 & 0 \\
\left(U_{n}\right)_{n=1}^{\infty} & 0
\end{array}\right]\left[\begin{array}{cc}
0 & \left(V_{n}\right)_{n=1}^{\infty} \\
0 & 0
\end{array}\right] .
$$

As $\left[\begin{array}{cc}{ }_{\left(U_{n}\right)_{n=1}^{\infty}}^{0} & 0\end{array}\right] \in P_{2} \mathfrak{A} P_{1}=P_{2} I P_{1} \subset I$ and $I$ is an ideal, it follows that $\left[\begin{array}{cc}0 & \left(S_{n}\right)_{n=1}^{\infty} \\ 0 & 0\end{array}\right] \in I$ as well. Since $\varepsilon>0$ was arbitrary, this entails that $\left(T_{n}\right)_{n=1}^{\infty} \in$ $P_{1} I P_{2}$, and since $\left(T_{n}\right)_{n=1}^{\infty} \in \ell^{\infty}\left(\mathcal{K}\left(\ell^{2}, \ell^{p}\right)\right)$ was arbitrary, this means that $P_{1} I P_{2}=P_{1} \mathfrak{A} P_{2}$.

Remarks. 1. Even though Proposition 5.4 shows that the (still hypothetical) non-amenability of $\ell^{\infty}\left(\mathcal{K}\left(\ell^{p} \oplus \ell^{2}\right)\right)$ cannot be established in the same way as for $\mathcal{B}\left(\ell^{p} \oplus \ell^{2}\right)$, it naturally leads to the question if, for sufficiently nice Banach spaces $E$, the amenability of $\ell^{\infty}(\mathcal{K}(E))$ forces $\mathcal{B}(E)$ to be amenable. Since $\mathcal{K}(E)^{* *}=\mathcal{B}(E)$ via trace duality for any reflexive Banach space with the approximation property, this question can, for such spaces, be put into a more general framework: If $\ell^{\infty}(\mathfrak{A})$ is amenable for some Banach algebra $\mathfrak{A}$, does this imply that $\mathfrak{A}^{* *}$, equipped with one of the Arens products (see [Dal]), is amenable? Partial answers, which do not apply to the case where $\mathfrak{A}=\mathcal{K}\left(\ell^{p} \oplus \ell^{2}\right)$, are given in [CS-R].

2. In [CS-R, Theorem 1], the following is claimed to be a consequence of [G-I]: For a unital $C^{*}$-algebra $\mathfrak{A}$, there are an index set $\mathbb{I}$, which can be chosen as $\mathbb{N}$ if $\mathfrak{A}^{*}$ is separable, and an algebra homomorphism from $\ell^{\infty}(\mathbb{I}, \mathfrak{A})$ onto $\mathfrak{A}^{* *}$. An inspection of the proof of [CS-R, Theorem 1] shows that the alleged algebra homomorphism is

$$
\ell^{\infty}(\mathbb{I}, \mathfrak{A}) \rightarrow \mathfrak{A}^{* *}, \quad\left(a_{i}\right)_{i \in \mathbb{I}} \mapsto \text { weak }^{*}-\lim _{i \in \mathcal{U}} a_{i}
$$


for a suitable ultrafilter $\mathcal{U}$ over $\mathbb{I}$. Let $\mathfrak{A}$ be the unitization of $\mathcal{K}\left(\ell^{2}\right)$, and let $\mathcal{U}$ be a free ultrafilter over $\mathbb{N}$. Then we have

$$
\text { weak }{ }^{*}-\lim _{n \in \mathcal{U}} \delta_{1} \odot \delta_{n}=\text { weak }^{*}-\lim _{n \in \mathcal{U}} \delta_{n} \odot \delta_{1}=0
$$

whereas

$$
\text { weak }{ }^{*}-\lim _{n \in \mathcal{U}}\left(\delta_{1} \odot \delta_{n}\right)\left(\delta_{n} \odot \delta_{1}\right)=\delta_{1} \odot \delta_{1} \neq 0,
$$

which means that (10) cannot be multiplicative, contrary to what is claimed in $[\mathrm{CS}-\mathrm{R}]$.

6. A left ideal in $\left(\mathcal{K}\left(\ell^{p}\right)\right) \mathcal{U}$ without bounded right approximate identity. If $\mathfrak{A}$ is an amenable Banach algebra, then a closed ideal of $\mathfrak{A}$ has a bounded approximate identity if and only if it is weakly complemented. By finding a closed, weakly complemented ideal of $\mathfrak{A}$ that lacks a bounded approximate identity, one can thus show that $\mathfrak{A}$ is not amenable, as was done in $[\mathrm{D}-\mathrm{G}-\mathrm{H}]$ in the case of the measure algebra of a non-discrete, locally compact group. More generally, a closed left ideal of $\mathfrak{A}$ has a bounded right approximate identity if and only if it is weakly complemented (see [Run, Lemma 2.3.6], for instance).

In this section, for $p \in(1,2)$ and a free ultrafilter $\mathcal{U}$ over $\mathbb{N}$, we shall exhibit a closed left ideal of $\left(\mathcal{K}\left(\ell^{p}\right)\right)_{\mathcal{U}}$ that lacks a right approximate identity (bounded or not) and present some, albeit circumstantial, evidence for it being weakly complemented.

For $p \in(1,2)$, let $\iota: \ell^{p} \rightarrow \ell^{2}$ be the natural inclusion map, and note that the adjoint $\iota^{*}: \ell^{2} \rightarrow \ell^{p^{\prime}}$ is the canonical inclusion of $\ell^{2}$ in $\ell^{p^{\prime}}$. Let $\mathcal{U}$ be a free ultrafilter over $\mathbb{N}$, and define

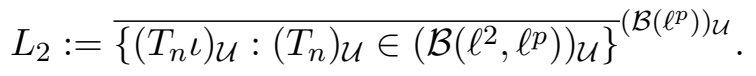

Obviously, $L_{2}$ is a closed left ideal of $\left(\mathcal{B}\left(\ell^{p}\right)\right) \mathcal{U}$. By Pitt's theorem ([L-T, Proposition 2.c.3]), we have $\mathcal{B}\left(\ell^{2}, \ell^{p}\right)=\mathcal{K}\left(\ell^{2}, \ell^{p}\right)$, so that $L_{2}$ is, in fact, a closed ideal of $\left(\mathcal{K}\left(\ell^{p}\right)\right)_{\mathcal{U}}$.

Recall that, for $\varepsilon>0$, a $(1+\varepsilon)$-isometry from a Banach space $E$ into a Banach space $F$ is a linear map $T: E \rightarrow F$ satisfying

$$
(1-\varepsilon)\|x\| \leq\|T x\| \leq(1+\varepsilon)\|x\| \quad(x \in E) .
$$

By [D-J-T, Dvoretzky's Theorem 19.1], there is, for each infinite-dimensional Banach space $E$, for each $n \in \mathbb{N}$, and for each $\varepsilon>0$, a $(1+\varepsilon)$-isometry from $\ell_{n}^{2}$ into $E$. We shall use this theorem to obtain particular elements of $L_{2}$. For each $n \in \mathbb{N}$, let $\pi_{n}: \ell^{2} \rightarrow \ell_{n}^{2}$ denote the canonical projection onto the first $n$ coordinates, and let, for each $n \in \mathbb{N}, \tau_{n}: \ell_{n}^{2} \rightarrow \ell^{p}$ be a $(1+1 / n)$-isometry, which exists by Dvoretzky's theorem. Then $\left(\tau_{n} \pi_{n} \iota\right)_{\mathcal{U}}$ lies in $L_{2}$.

The following is our technical main result in this section: 
Lemma 6.1. Let $p \in(1,2)$, let $\mathcal{U}$ be a free ultrafilter over $\mathbb{N}$, and let $L_{2}$ and $\left(\tau_{n} \pi_{n} \iota\right)_{\mathcal{U}}$ be defined as above. Then

$$
\left\|\left(\tau_{n} \pi_{n} \iota\right)_{\mathcal{U}}\left(T_{n}\right)_{\mathcal{U}}-\left(\tau_{n} \pi_{n} \iota\right)_{\mathcal{U}}\right\| \geq 1 \quad\left(\left(T_{n}\right)_{\mathcal{U}} \in L_{2}\right) .
$$

Proof. Assume towards a contradiction that there are $\theta \in[0,1)$ and $\left(T_{n}\right)_{\mathcal{U}} \in\left(\mathcal{B}\left(\ell^{2}, \ell^{p}\right)\right)_{\mathcal{U}}$ such that

$$
\begin{aligned}
\theta & >\left\|\left(\tau_{n} \pi_{n} \iota\right)_{\mathcal{U}}\left(T_{n} \iota\right)_{\mathcal{U}}-\left(\tau_{n} \pi_{n} \iota\right)_{\mathcal{U}}\right\| \\
& =\lim _{n \in \mathcal{U}}\left\|\tau_{n} \pi_{n} \iota T_{n} \iota-\tau_{n} \pi_{n} \iota\right\|=\lim _{n \in \mathcal{U}}\left\|\pi_{n} \iota T_{n} \iota-\pi_{n} \iota\right\|,
\end{aligned}
$$

where the last equality is due to the fact that $\tau_{n}$ is a $(1+1 / n)$-isometry for each $n \in \mathbb{N}$.

Let $T:=$ weak- $\lim _{n \in \mathcal{U}} T_{n} \in \mathcal{K}\left(\ell^{2}, \ell^{p}\right)$. (The limit exists by [Hei, Propositon 1.45] because $\mathcal{K}\left(\ell^{2}, \ell^{p}\right)$ is reflexive, so that its closed unit ball is weakly compact.) Let $x \in \ell^{p}$ and $\xi \in \ell^{2}$. Then

$$
\begin{aligned}
\mid\langle\xi,(\iota T \iota-\iota) & (x)\rangle \mid \\
& =\left|\left\langle\iota^{*}(\xi),(T \iota)(x)\right\rangle-\langle\xi, \iota(x)\rangle\right| \\
& =\lim _{n \in \mathcal{U}}\left|\left\langle\iota^{*}(\xi),\left(T_{n} \iota\right)(x)\right\rangle-\langle\xi, \iota(x)\rangle\right|=\lim _{n \in \mathcal{U}}\left|\left\langle\xi,\left(\iota T_{n} \iota-\iota\right)(x)\right\rangle\right| \\
& \left.=\lim _{n \in \mathcal{U}}\left|\left\langle\pi_{n}^{*}(\xi),\left(\iota T_{n} \iota-\iota\right)(x)\right\rangle\right| \quad \text { (because } \lim _{n \rightarrow \infty}\left\|\pi_{n}^{*}(\xi)-\xi\right\|_{2}=0\right) \\
& =\lim _{n \in \mathcal{U}}\left|\left\langle\xi,\left(\pi_{n} \iota T_{n} \iota-\pi_{n} \iota\right)(x)\right\rangle\right| \leq \lim _{n \in \mathcal{U}}\left\|\pi_{n} \iota T_{n} \iota-\pi_{n} \iota\right\|\|x\|\|\xi\| \\
& <\theta\|x\|\|\xi\| \quad(\text { by }(12)) .
\end{aligned}
$$

It follows that

$$
\|\iota T \iota-\iota\| \leq \theta .
$$

Since $T$ is compact, so is $\iota T \iota$. Hence, there is a strictly increasing sequence $\left(n_{k}\right)_{k=1}^{\infty}$ in $\mathbb{N}$ such that $\left((\iota T \iota)\left(\delta_{n_{k}}\right)\right)_{k=1}^{\infty}$ is norm convergent in $\ell^{2}$ with limit $\eta$, say. It follows that

$$
\lim _{k \rightarrow \infty}\left\langle\delta_{n_{k}},(\iota T \iota)\left(\delta_{n_{k}}\right)\right\rangle=\lim _{k \rightarrow \infty}\left\langle\delta_{n_{k}}, \eta\right\rangle=0 .
$$

Together, (13) and (14) yield

$$
\begin{aligned}
1 & =\lim _{k \rightarrow \infty}\left\langle\delta_{n_{k}}, \iota\left(\delta_{n_{k}}\right)\right\rangle \\
& =\lim _{k \rightarrow \infty}\left|\left\langle\delta_{n_{k}},(\iota T \iota)\left(\delta_{n_{k}}\right)\right\rangle-\left\langle\delta_{n_{k}}, \iota\left(\delta_{n_{k}}\right)\right\rangle\right|=\lim _{k \rightarrow \infty}\left|\left\langle\delta_{n_{k}},(\iota T \iota-\iota)\left(\delta_{n_{k}}\right)\right\rangle\right| \leq \theta,
\end{aligned}
$$

which is impossible because $\theta \in[0,1)$.

The following is now immediate:

Proposition 6.2. Let $p \in(1,2)$, let $\mathcal{U}$ be a free ultrafilter over $\mathbb{N}$, and let $L_{2}$ be the closed left ideal of $\left(\mathcal{K}\left(\ell^{p}\right)\right) \mathcal{U}$ defined in (11). Then $L_{2}$ does not have a right approximate identity. 
REMARK. Both Lemma 6.1 and Proposition 6.2 remain true in the slightly more general situation where $\mathcal{U}$ is a countably incomplete ultrafilter over an arbitrary index set.

If we could establish that $L_{2}$ is weakly complemented, i.e., has a complemented annihilator in $\left(\mathcal{K}\left(\ell^{p}\right)\right)_{\mathcal{U}}^{*}$, then we would know that $\left(\mathcal{K}\left(\ell^{p}\right)\right) \mathcal{U}$ - and thus, by Theorem $2.1, \mathcal{B}\left(\ell^{p}\right)$ - cannot be amenable. Unfortunately, such a proof eludes us, mostly due to the lack of a suitable description of $\left(\mathcal{K}\left(\ell^{p}\right)\right)_{\mathcal{U}}^{*}$. Nevertheless, we are able to show that the annihilator of $L_{2}$ in a certain closed subspace of $\left(\mathcal{K}\left(\ell^{p}\right)\right)_{\mathcal{U}}^{*}$ is indeed complemented.

We achieve this as a by-product of a general complementation result for ultrapowers of vector-valued $\ell^{p}$-spaces.

Given a set $S$ and an ultrafilter $\mathcal{U}$ over some index set $\mathbb{I}$, we use $\langle S\rangle_{\mathcal{U}}$ for the corresponding set-theoretic ultrapower (see [Hei] for the definition). For $\left(s_{i}\right)_{i \in \mathbb{I}} \in S^{\mathbb{I}}$, we denote its image in $\langle S\rangle_{\mathcal{U}}$ by $\left\langle s_{i}\right\rangle_{\mathcal{U}}$.

The following lemma relates the spaces $\ell^{p}\left(\langle\mathbb{N}\rangle_{\mathcal{U}},(E)_{\mathcal{U}}\right)$ and $\left(\ell^{p}(E)\right)_{\mathcal{U}}$ for $p \in[1, \infty$ ), a Banach space $E$, and an ultrafilter $\mathcal{U}$ (over an arbitrary index set). We identify the finitely supported functions in $\ell^{p}\left(\langle\mathbb{N}\rangle_{\mathcal{U}},(E)_{\mathcal{U}}\right)$ with the algebraic tensor product $c_{00}\left(\langle\mathbb{N}\rangle_{\mathcal{U}}\right) \otimes(E)_{\mathcal{U}}$, where $c_{00}\left(\langle\mathbb{N}\rangle_{\mathcal{U}}\right)$ are the finitely supported functions from $\langle\mathbb{N}\rangle_{\mathcal{U}}$ into $\mathbb{C}$.

Lemma 6.3. Let $p \in[1, \infty)$, let $E$ be a Banach space, and let $\mathcal{U}$ be an ultrafilter. Then there is a unique isometry $J_{p}: \ell^{p}\left(\langle\mathbb{N}\rangle_{\mathcal{U}},(E)_{\mathcal{U}}\right) \rightarrow\left(\ell^{p}(E)\right)_{\mathcal{U}}$ given by

$$
J_{p}\left(\delta_{\left\langle n_{i}\right\rangle_{\mathcal{U}}} \otimes\left(x_{i}\right)_{\mathcal{U}}\right)=\left(\delta_{n_{i}} \otimes x_{i}\right)_{\mathcal{U}} \quad\left(\delta_{\left\langle n_{i}\right\rangle_{\mathcal{U}}} \in\langle\mathbb{N}\rangle_{\mathcal{U}},\left(x_{i}\right)_{\mathcal{U}} \in(E)_{\mathcal{U}}\right) .
$$

Proof. It is routinely checked that $(15)$ defines an isometry from $c_{00}\left(\langle\mathbb{N}\rangle_{\mathcal{U}}\right)$ $\otimes(E)_{\mathcal{U}}$ into $\left(\ell^{p}(E)\right)_{\mathcal{U}}$, which then extends to all of $\ell^{p}\left(\langle\mathbb{N}\rangle_{\mathcal{U}},(E)_{\mathcal{U}}\right)$ by continuity.

Lemma 6.3 enables us to canonically identify $\ell^{p}\left(\langle\mathbb{N}\rangle_{\mathcal{U}},(E)_{\mathcal{U}}\right)$ with a closed subspace of $\left(\ell^{p}(E)\right) \mathcal{U}$.

Given a Banach space $E$ and an ultrafilter $\mathcal{U}$, there is a canonical duality between $(E)_{\mathcal{U}}$ and $\left(E^{*}\right)_{\mathcal{U}}$, which induces an isometric embedding of $\left(E^{*}\right)_{\mathcal{U}}$ into $(E)_{\mathcal{U}}^{*}$; for countably incomplete $\mathcal{U}$, this embedding is an isomorphism if and only if $(E)_{\mathcal{U}}$ is reflexive ([Hei, Proposition 7.1]). Recall that $E$ is called superreflexive if every Banach space that can be finitely represented in $E$ is reflexive; equivalently, $E$ is superreflexive if and only if $(E)_{\mathcal{U}}$ is reflexive for each ultrafilter $\mathcal{U}$ ([Hei, Proposition 6.4]). Also, if $E$ is superreflexive and $p \in(1, \infty)$, then $\ell^{p}(E)$ is superreflexive as well ([Daw 1, Proposition 4]). All this guarantees that the map $\Pi_{p}$ in the following proposition is well defined.

Proposition 6.4. Let $p \in(1, \infty)$, let $E$ be a superreflexive Banach space, let $\mathcal{U}$ be an ultrafilter, and let $J_{p}: \ell^{p}\left(\langle\mathbb{N}\rangle_{\mathcal{U}},(E)_{\mathcal{U}}\right) \rightarrow\left(\ell^{p}(E)\right)_{\mathcal{U}}$ and 
$J_{p^{\prime}}: \ell^{p^{\prime}}\left(\langle\mathbb{N}\rangle_{\mathcal{U}},\left(E^{*}\right)_{\mathcal{U}}\right) \rightarrow\left(\ell^{p^{\prime}}\left(E^{*}\right)\right)_{\mathcal{U}}$ be as in Lemma 6.3. Then $\Pi_{p}:=J_{p} J_{p^{\prime}}^{*}$ is a norm one projection onto $\ell^{p}\left(\langle\mathbb{N}\rangle_{\mathcal{U}},(E)_{\mathcal{U}}\right)$. Moreover, for any $q \in(p, \infty]$ we have

$$
\operatorname{ker} \Pi_{p}=\left\{\left(x_{i}\right)_{\mathcal{U}} \in\left(\ell^{p}(E)\right)_{\mathcal{U}}: \lim _{i \in \mathcal{U}}\left\|x_{i}\right\|_{\ell^{q}(E)}=0\right\} .
$$

Proof. It is easy to see that $\Pi_{p}$ is indeed a norm one projection onto $\ell^{p}\left(\langle\mathbb{N}\rangle_{\mathcal{U}},(E)_{\mathcal{U}}\right)$.

Let $\mathbb{I}$ be the index set over which $\mathcal{U}$ is defined. For $\left(n_{i}\right)_{i \in \mathbb{I}} \in \mathbb{N}^{\mathbb{I}}$, let $P_{n_{i}}: \ell^{p}(E) \rightarrow E$ denote the projection onto the $n_{i}$ th coordinate. From the definition of $\Pi_{p}$, it is clear that $\left(x_{i}\right)_{\mathcal{U}} \in\left(\ell^{p}(E)\right)_{\mathcal{U}}$ belongs to ker $\Pi_{p}$ if and only if $\lim _{i \in \mathcal{U}}\left\|P_{n_{i}} x_{i}\right\|_{E}=0$ for any $\left(n_{i}\right)_{i \in \mathbb{I}} \in \mathbb{N}^{\mathbb{I}}$. It follows that

$$
\operatorname{ker} \Pi_{p} \supset\left\{\left(x_{i}\right)_{\mathcal{U}} \in\left(\ell^{p}(E)\right)_{\mathcal{U}}: \lim _{i \in \mathcal{U}}\left\|x_{i}\right\|_{\ell^{\infty}(E)}=0\right\}
$$

For the converse inclusion, let $\left(x_{i}\right)_{\mathcal{U}} \in\left(\ell^{p}(E)\right)_{\mathcal{U}}$ be such that $\lim _{i \in \mathcal{U}}\left\|x_{i}\right\|_{\ell^{\infty}(E)}$ $=: \delta>0$. Let $U \in \mathcal{U}$ be such that $\left\|x_{i}\right\|_{\ell^{\infty}(E)}=\sup _{n \in \mathbb{N}}\left\|P_{n} x_{i}\right\|>\delta / 2$ for each $i \in U$. For each $i \in U$, choose $n_{i} \in \mathbb{N}$ such that $\left\|P_{n_{i}} x_{i}\right\|_{\ell^{\infty}(E)}>\delta / 2$. It follows that $\lim _{i \in \mathcal{U}}\left\|P_{n_{i}} x_{i}\right\|_{\ell^{\infty}(E)} \geq \delta / 2>0$, whence $\left(x_{i}\right)_{\mathcal{U}} \in\left(\ell^{p}(E)\right)_{\mathcal{U}} \notin \operatorname{ker} \Pi_{p}$. All in all, we have

$$
\operatorname{ker} \Pi_{p}=\left\{\left(x_{i}\right)_{\mathcal{U}} \in\left(\ell^{p}(E)\right)_{\mathcal{U}}: \lim _{i \in \mathcal{U}}\left\|x_{i}\right\|_{\ell^{\infty}(E)}=0\right\} .
$$

Let $q \in(p, \infty)$. In view of $(17)$, it is clear that

$$
\operatorname{ker} \Pi_{p} \supset\left\{\left(x_{i}\right)_{\mathcal{U}} \in\left(\ell^{p}(E)\right)_{\mathcal{U}}: \lim _{i \in \mathcal{U}}\left\|x_{i}\right\|_{\ell^{q}(E)}=0\right\} .
$$

For the converse inclusion, note that, for any $x=\left(x_{n}\right)_{n=1}^{\infty} \in \ell^{p}(E)$, we have

$$
\begin{aligned}
\|x\|_{\ell^{q}(E)}^{q} & =\sum_{n=1}^{\infty}\left\|x_{n}\right\|^{q}=\sum_{n=1}^{\infty}\left\|x_{n}\right\|^{q-p}\left\|x_{n}\right\|^{p} \leq\|x\|_{\ell^{\infty}(E)}^{q-p} \sum_{n=1}^{\infty}\left\|x_{n}\right\|^{p} \\
& \leq\|x\|_{\ell^{\infty}(E)}^{q-p}\|x\|_{\ell^{p}(E)}^{p} .
\end{aligned}
$$

Consequently, if $\left(x_{i}\right)_{\mathcal{U}} \in \operatorname{ker} \Pi_{p}$, that is, if $\lim _{i \in \mathcal{U}}\left\|x_{i}\right\|_{\ell^{\infty}(E)}=0$, then $\lim _{i \in \mathcal{U}}\left\|x_{i}\right\|_{\ell^{q}(E)}=0$ as well. This proves (16).

Let $p \in(1,2)$, and let $\mathcal{U}$ be a free ultrafilter over $\mathbb{N}$. We can canonically represent $\left(\mathcal{B}\left(\ell^{p}\right)\right)_{\mathcal{U}}$ on $\left(\ell^{p}\right) \mathcal{U}$ by letting

$$
\left(T_{n}\right)_{\mathcal{U}}\left(x_{n}\right)_{\mathcal{U}}=\left(T_{n} x_{n}\right)_{\mathcal{U}} \quad\left(\left(T_{n}\right)_{\mathcal{U}} \in\left(\mathcal{B}\left(\ell^{p}\right)\right)_{\mathcal{U}},\left(x_{n}\right)_{\mathcal{U}} \in\left(\ell^{p}\right)_{\mathcal{U}}\right)
$$

Clearly, $\left(T_{n}\right) \mathcal{U}\left(x_{n}\right) \mathcal{U}=0$ holds for all $\left(T_{n}\right)_{\mathcal{U}} \in L_{2}$ if and only if $\lim _{n \in \mathcal{U}}\left\|x_{n}\right\|_{2}$ $=0$, i.e., $\left(x_{n}\right)_{\mathcal{U}} \in \operatorname{ker} \Pi_{p}$ by Proposition 6.4.

The Banach space $\mathcal{B}\left(\left(\ell^{p}\right) \mathcal{U}\right)$ has the canonical predual $\left(\ell^{p}\right) \mathcal{U} \hat{\otimes}\left(\ell^{p^{\prime}}\right) \mathcal{U}$, which, by [Daw 2, Proposition 4.7], embeds isometrically into $\left(\ell^{p} \hat{\otimes} \ell^{p^{\prime}}\right) \mathcal{U}=$ $\left(\mathcal{K}\left(\ell^{p}\right)^{*}\right) \mathcal{U}$ and thus into $\left(\mathcal{K}\left(\ell^{p}\right)\right)_{\mathcal{U}}^{*}$ (see [Hei, p. 87]). It therefore makes sense to speak of the annihilator of $L_{2}$ in $\left(\ell^{p}\right) \mathcal{U} \hat{\otimes}\left(\ell^{p^{\prime}}\right) \mathcal{U}$.

In view of the foregoing we have: 
Corollary 6.5. Let $p \in(1,2)$, and let $\mathcal{U}$ be a free ultrafilter. Then the annihilator of $L_{2}$ in $\left(\ell^{p}\right) \mathcal{U} \hat{\otimes}\left(\ell^{p^{\prime}}\right) \mathcal{U}$ is its complemented subspace $\operatorname{ker} \Pi_{p} \hat{\otimes}$ $\left(\ell^{p^{\prime}}\right) \mathcal{U}$, where $\Pi_{p}$ is the canonical projection from $\left(\ell^{p}\right)_{\mathcal{U}}$ onto $\ell^{p}\left(\langle\mathbb{N}\rangle_{\mathcal{U}}\right)$.

REMARK. It would be interesting to know whether the annihilator of $L_{2}$ in $\left(\mathcal{K}\left(\ell^{p}\right)^{*}\right) \mathcal{U}$ is complemented: as $\left(\mathcal{K}\left(\ell^{p}\right)\right)_{\mathcal{U}}^{*}$ can be finitely represented in $\left(\mathcal{K}\left(\ell^{p}\right)^{*}\right) \mathcal{U}$ ([Hei, Theorem 7.3]), this would further support our belief that $L_{2}$ is weakly complemented.

\section{References}

[CS-R] F. Cabello Sánchez and R. García, On amenability of the Banach algebras $\ell_{\infty}(S, \mathfrak{A})$, Math. Proc. Cambridge Philos. Soc. 132 (2002), 319-322.

[Cun] J. Cuntz, Simple $C^{*}$-algebras generated by isometries, Comm. Math. Phys. 57 (1977), 173-185.

[Dal] H. G. Dales, Banach Algebras and Automatic Continuity, London Math. Soc. Monogr. (N.S.) 24, Clarendon Press, Oxford, 2000.

[D-G-H] H. G. Dales, F. Ghahramani, and A. Ya. Helemskiı̌, The amenability of measure algebras, J. London Math. Soc. (2) 66 (2002), 213-226.

[Daw 1] M. Daws, Arens regularity of the algebra of operators on a Banach space, Bull. London Math. Soc. 36 (2004), 493-503.

[Daw 2] -, Amenability of ultraproducts of Banach algebras, Proc. Edinburgh Math. Soc., to appear.

[D-F] A. Defant and K. Floret, Tensor Norms and Operator Ideals, North-Holland Math. Stud. 176, North-Holland, Amsterdam, 1993.

[D-J-T] J. Diestel, H. Jarchow, and A. Tonge, Absolutely Summing Operators, Cambridge Stud. Adv. Math. 43, Cambridge Univ. Press, Cambridge, 1995.

[G-I] G. Godefroy and B. Iochum, Arens-regularity of Banach algebras and the geometry of Banach spaces, J. Funct. Anal. 80, (1988), 47-59.

[Grø] N. Grønbæk, Various notions of amenability, a survey of problems, in: E. Albrecht and M. Mathieu (eds.), Banach Algebras '97, de Gruyter, Berlin, 1998, 535-547.

[G-J-W] N. Grønbæk, B. E. Johnson, and G. A. Willis, Amenability of Banach algebras of compact operators, Israel J. Math. 87 (1994), 289-324.

[Hei] S. Heinrich, Ultraproducts in Banach space theory, J. Reine Angew. Math. 313 (1980), 72-104.

[Joh 1] B. E. Johnson, Cohomology in Banach algebras, Mem. Amer. Math. Soc. 127 (1972).

[Joh 2] - Approximate diagonals and cohomology of certain annihilator Banach algebras, Amer. J. Math. 94 (1972), 685-698.

[Joh 3] -, Symmetric amenability and the nonexistence of Lie and Jordan derivations, Math. Proc. Cambridge Philos. Soc. 120 (1996), 455-473.

[L-L-W] A. T.-M. Lau, R. J. Loy, and G. A. Willis, Amenability of Banach and $C^{*}$ algebras on locally compact groups, Studia Math. 119 (1996), 161-178.

[L-P] J. Lindenstrauss and A. Pełczyński, Absolutely summing operators in $\mathcal{L}_{p}$-spaces and their applications, ibid. 29 (1968), 275-326.

[L-R] J. Lindenstrauss and H. P. Rosenthal, The $\mathcal{L}_{p}$ spaces, Israel J. Math. 7 (1969), 325-349. 
[L-T] J. Lindenstrauss and L. Tzafriri, Classical Banach Spaces, I. Ergeb. Math. Grenzgeb. 92, Springer, Berlin, 1977.

[Oza] N. Ozawa, $A$ note on non-amenability of $\mathcal{B}\left(l_{p}\right)$ for $p=1,2$, Int. J. Math. 15 (2004), 557-565.

[Pat] A. L. T. Paterson, Amenability, Math. Surveys Monogr. 29, Amer. Math. Soc., Providence, RI, 1988.

[Pie] A. Pietsch, Operator Ideals, North-Holland Math. Library 20, North-Holland, Amsterdam, 1980.

[Pis] G. Pisier, On Read's proof that $B\left(l_{1}\right)$ is not amenable, in: Geometric Aspects of Functional Analysis, Lecture Notes in Math. 1850, Springer, Berlin, 2004, 269-275.

[Rea] C. J. Read, Relative amenability and the non-amenability of $B\left(l^{1}\right)$, J. Austral. Math. Soc. 80 (2006), 317-333.

[Run] V. Runde, Lectures on Amenability, Lecture Notes in Math. 1774, Springer, Berlin, 2002.

[Sim] B. Sims, "Ultra"-Techniques in Banach Space Theory, Queen's Papers in Pure Appl. Math. 60, Queen's Univ., Kingston, ON, 1982.

[Tak] M. Takesaki, Theory of Operator Algebras, III, Encyclopaedia Math. Sci. 127, Springer, Berlin, 2003.

[T-J] N. Tomczak-Jaegermann, Banach-Mazur Distances and Finite-Dimensional Operator Ideals, Pitman Monogr. Surveys Pure Appl. Math. 38, Longman Sci. \& Tech., Harlow, 1989.

[Was] S. Wassermann, On tensor products of certain group $C^{*}$-algebras, J. Funct. Anal. 23 (1976), 239-254.

Department of Pure Mathematics

University of Leeds

Department of Mathematical and Statistical Sciences

Leeds, LS2 9JT, United Kingdom

University of Alberta

E-mail:matt.daws@cantab.net Edmonton, AB, Canada T6G 2G1

E-mail: vrunde@ualberta.ca

Received November 27, 2007

Revised version June 8, 2008 